\title{
Synthesis of New Di- and Triamides as Potential Organocatalysts for Asymmetric Aldol Reaction in Water
}

\author{
Elif Keskin, Cigdem Yolacan and Feray Aydogan* \\ Department of Chemistry, Yildiz Technical University, Davutpasa Campus, 34010 Esenler, Istanbul, Turkey \\ *Corresponding author: E-mail: feray_aydogan@yahoo.com \\ Tel: +902123834215
}

Received: 09-27-2018

\begin{abstract}
New di- or triamide organocatalysts derived from $(L)$-proline were synthesized and successfully used in the direct asymmetric aldol reaction of aliphatic ketones and aromatic aldehydes in water at $0{ }^{\circ} \mathrm{C}$ in the presence of benzoic acid as co-catalyst. (S)-methyl-2-((S)-3-hydroxy-2-((S)-3-pyrrolidine-2-carboxamido)propanamido)-3-phenylpropanoate (7c) as organocatalyst showed best results under these reaction conditions, and good diastereoselectivities (up to $99 \%$ ), enantioselectivities (up to 98\%) and yields (up to 91\%) were observed.
\end{abstract}

Keywords: Aldol reaction; organocatalysis; asymmetric synthesis; prolinamide

\section{Introduction}

The aldol reaction is an important tool in organic chemistry to synthesize attractive intermediates. This carbon-carbon bond formation reaction yields the $\beta$-hydroxyketones which are important precursors for pharma- ceuticals and natural products. Especially, the asymmetric version of the reaction has been extensively studied and used for the synthesis of valuable intermediates, highly functionalized and complex molecules with important biological activities. The most considerable asymmetric method is the using of asymmetric organocatalysis which<smiles>C[C@H](NC(=O)c1ccccc1NC(=O)C1CCCN1)c1ccccc1</smiles><smiles>C[C@H](NC(=O)c1ccccc1NC(=O)C1CCCN1)c1ccccc1</smiles><smiles>CCCCNC(=O)c1ccccc1NC(=O)C1CCCN1</smiles><smiles>O=C(NC1CCCCC1)c1ccccc1NC(=O)C1CCCN1</smiles><smiles>COC(=O)[C@H](CO)NC(=O)[C@H](Cc1ccccc1)NC(=O)C1CCCN1</smiles>

$7 \mathbf{a}$<smiles>CCCCNC(=O)c1ccccc1NC(=O)C(Cc1ccccc1)NC(=O)C1CCCN1</smiles><smiles>COC(=O)[C@H](Cc1ccccc1)NC(=O)[C@H](CO)NC(=O)C1CCCN1</smiles>

7c

Figure 1. The structures of organocatalysts investigated in this study 
are non-metal, small, easily synthesizable organic compounds with low toxicity and mostly natural products. ${ }^{1-3}$ Among these natural organocatalysts, $L$-proline is the corner stone of natural amino acid organocatalysts and used successfully to catalyze various organic reactions. ${ }^{4-7}$ But, this small organic compound has some drawbacks such as low solubility, low yields and low enantioselectivity with aromatic aldehydes as organocatalyst in asymmetric aldol condensation. ${ }^{8}$ To overcome these drawbacks, some derivatives of proline had been succesfully synthesized and used in asymmetric aldol reaction and new modifications on proline are still under investigation. ${ }^{9-24}$ Especially, proline based amides have been used succesfully for this reaction. ${ }^{25-30}$ These derivatives have some advantages such as easy preperation, high stability and the presence of important functional groups. The catalytic effect of these catalysts based on secondary amine group on the pyrrolidine ring which form enamine to activate carbonyl group and the hyrogen bond donors which improve the activation of electrophile and selectivity. These advantages make them one of the most popular organocatalysts for organic syn- thesis and the design and investigation of new proline based amides as organocatalysts for various organic reactions is still under investigation by several research groups. $^{31,32}$

In our continuing research on the synthesis of chiral organocatalysts and their investigation in direct asymmetric aldol reaction, we have investigated the catalytic potential of some proline amide derivatives and also 1,2,3,4-tetrahydroisoquinoline and thiazolidine-4-carboxylic acid amide derivatives. ${ }^{33-36}$ Now we herein report the synthesis of new proline based amides (3a-d and $7 \mathbf{a}-$ c) (Figure 1) and their application in asymmetric direct aldol reaction.

\section{Results and Discussion}

The reaction of Boc-protected $L$-proline with aminobenzamide derivatives (1a-d) which were synthesized by the reaction of isatoic anhydride with some amines and subsequent deprotection of products (2a-d) gave new pro-

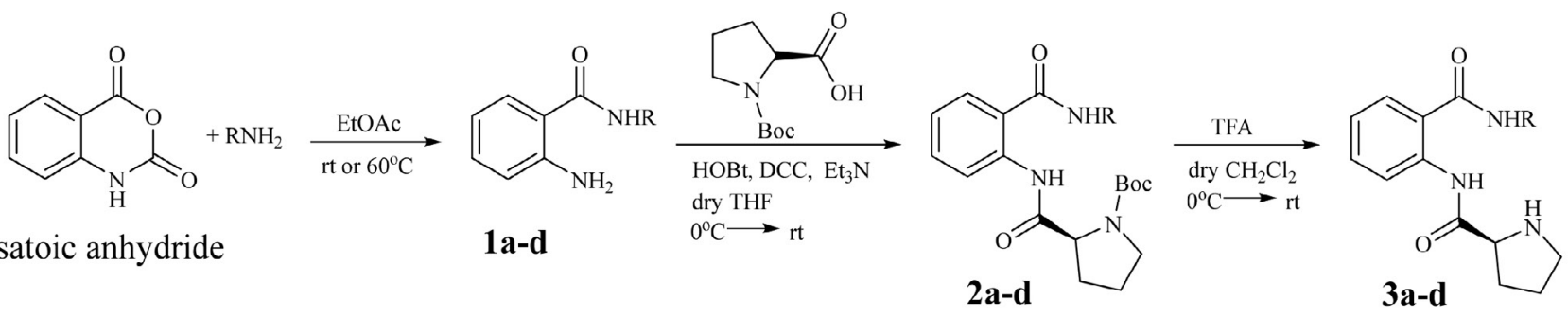

Scheme 1. The synthetic route for compounds $3 \mathbf{a}-\mathbf{d}$.<smiles>O=C(O)[C@@H]1CCCN1C(=O)O</smiles><smiles>[R]C(N)C(=O)OC</smiles><smiles>[R]NC(=O)C([R])NC(=O)C1CCCN1</smiles><smiles>CC(C)C1CCCCC1</smiles>
$0^{\circ} \mathrm{C} \longrightarrow \mathrm{rt}$

4a and $4 c$<smiles>[R]C(NC(=O)[C@@H]1CCCN1C(=O)c1ccccc1)C(=O)OC</smiles>

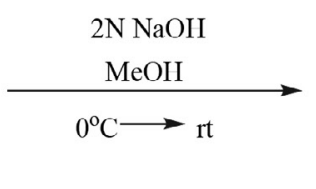

$\mid$\begin{tabular}{l|l}
$\mathrm{R}^{\prime} \mathrm{NH}_{2}$ \\
$\mathrm{IIOBt}, \mathrm{DCC}$ \\
$\mathrm{Et}_{3} \mathrm{~N}$, dry THF \\
$\mathrm{O}^{\circ} \mathrm{C} \longrightarrow{ }_{\mathrm{rt}}$
\end{tabular}
$5 \mathrm{a}$ and $5 \mathrm{c}$<smiles>[R]C(NC(=O)[C@@H]1CCCN1C(=O)O)C(=O)O</smiles><smiles>[R]NC(=O)[C@@H]([Z])NC(=O)[C@@H]1CCCN1C(=O)[O-]</smiles>

Scheme 2. The synthetic route for compounds $7 \mathbf{a}-\mathbf{c}$. 
line based diamides (3a-d) (Scheme 1). Aminobenzamides (1a-d) were known in the literature ${ }^{37,38}$ and the IR, ${ }^{1} \mathrm{H}$ NMR and MS data of the compounds 1a-d were in accordance with those data. The characterization of unknown compounds $\mathbf{2 a - d}$ and $\mathbf{3 a} \mathbf{a}-\mathbf{d}$ were performed from their spectral data.

Table 1. Catalytic activities of di- and tri-amides.

\begin{tabular}{|c|c|c|c|c|c|}
\hline \multirow[b]{3}{*}{$\begin{array}{l}\text { Catalyst } \\
(10 \mathrm{~mol} \%)\end{array}$} & \multirow[b]{3}{*}{ Solvent } & \multirow{2}{*}{\multicolumn{2}{|c|}{$\begin{array}{c}\begin{array}{c}\text { catalyst 3a-d } \\
\text { and 7a-c } \\
(10 \mathrm{~mol} \%)\end{array} \\
\text { benzoic acid }(10 \mathrm{~m}\end{array}$}} & & \multirow[b]{3}{*}{$\begin{array}{c}\text { Yield }^{b} \\
(\%)\end{array}$} \\
\hline & & & & & \\
\hline & & $\begin{array}{l}\text { Reaction } \\
\text { time }(h)\end{array}$ & $\begin{array}{c}\mathrm{dr}^{\mathrm{a}} \\
\text { (syn/anti) }\end{array}$ & $\begin{array}{c}\mathrm{ee}^{\mathrm{a}}(\%) \\
\text { (anti) }\end{array}$ & \\
\hline $3 a$ & DCM & 72 & $1 / 1.7$ & 16.7 & 84.2 \\
\hline $3 a$ & $\mathrm{H}_{2} \mathrm{O}$ & 24 & $1 / 2.2$ & 32.0 & 79.9 \\
\hline $3 a$ & none & 48 & $1 / 1.5$ & 32.4 & 77.7 \\
\hline $3 \mathbf{b}$ & DCM & 72 & $1 / 1.7$ & 21.6 & 58.8 \\
\hline $3 \mathbf{b}$ & $\mathrm{H}_{2} \mathrm{O}$ & 24 & $1 / 2.6$ & 42.4 & 83.0 \\
\hline $3 c$ & DCM & 72 & $1 / 2.3$ & 24.7 & 58.4 \\
\hline $3 c$ & $\mathrm{H}_{2} \mathrm{O}$ & 72 & $1 / 1.8$ & 34.5 & 75.7 \\
\hline $3 c$ & none & 48 & $1 / 1.7$ & 34.9 & 83.1 \\
\hline $3 d$ & DCM & 72 & $1 / 2.1$ & 28.8 & 80.6 \\
\hline $3 d$ & $\mathrm{H}_{2} \mathrm{O}$ & 24 & $1 / 1.9$ & 45.9 & 95.8 \\
\hline $3 d$ & none & 48 & $1 / 1.6$ & 29.0 & 86.2 \\
\hline $7 \mathbf{a}$ & DCM & 72 & $1 / 2.7$ & 47.9 & 86.9 \\
\hline $7 \mathbf{a}$ & $\mathrm{H}_{2} \mathrm{O}$ & 24 & $1 / 3.9$ & 65.5 & 70.3 \\
\hline $7 \mathbf{a}$ & none & 24 & $1 / 1.6$ & 68.3 & 63.7 \\
\hline $7 \mathbf{b}$ & DCM & 72 & $1 / 3.5$ & 50.7 & 76.0 \\
\hline $7 \mathbf{b}$ & $\mathrm{H}_{2} \mathrm{O}$ & 24 & $1 / 2.5$ & 32.0 & 91.0 \\
\hline $7 \mathbf{b}$ & none & 48 & $1 / 2.7$ & 53.3 & 96.0 \\
\hline $7 \mathrm{c}$ & DCM & 72 & $1 / 3.9$ & 78.5 & 52.0 \\
\hline $7 \mathrm{c}$ & $\mathrm{H}_{2} \mathrm{O}$ & 48 & $1 / 9.3$ & 90.6 & 89.4 \\
\hline $7 \mathrm{c}$ & none & 72 & $1 / 4.7$ & 87.5 & 96.0 \\
\hline $7 \mathrm{c}$ & $\mathrm{H}_{2} \mathrm{O} / \mathrm{THF}(2 / 1)$ & 72 & $1 / 5.6$ & 89.4 & 88.1 \\
\hline
\end{tabular}

${ }^{a}$ Determined by chiral-phase HPLC analysis. ${ }^{b}$ Combined yields of isolated diastereomers.
Compounds $7 \mathrm{a}-\mathrm{c}$ were synthesized by a reaction sequence as amidation, hydrolysis, second amidation and deprotection reactions starting from Boc-protected proline and some amino acid esters and amine (Scheme 2). All new compounds were in accordance with their spectral data.

The reaction of $p$-nitrobenzaldehyde with cyclohexanone under diferent conditions was chosen as a model reaction to investigate the catalytic activities of amide compounds 3 a-d and $7 \mathbf{a}-\mathbf{c}$, in direct asymmetric aldol reactions. First, the reaction was carried out with new amide compounds in water or dichloromethane (DCM), or without any solvent in the presence of benzoic acid (BA) as co-catalyst at room temperature. The results are shown in the Table 1. Compound $7 \mathbf{c}$ showed the best catalytic activity with good diastereoselectivity (90\%), enantioselectivity (91\%) and yield (89\%). The poor activities of $\mathbf{3}$ a-d can be interpreted that the planar phenyl rings prevent the appropriate arrangement in the transition state due to the sterical effect and thus these compounds did not show good asymmetric induction. Compounds $7 \mathbf{a}$ and $7 \mathbf{b}$ also showed lower selectivities due to the steric effect of phenyl group. The best asymmetric induction was obtained with $7 \mathrm{c}$, which has less steric effect around proline $\mathrm{NH}$ and prolinamide $\mathrm{NH}$. It is also thought that the $\mathrm{OH}$ group is effective in asymmetric induction through hydrogen bond formation.

Then, various co-catalysts containing 4-nitrobenzoic acid (4-NBA), $(2 R, 3 R)-(+)$-tartaric acid $(2 R, 3 R-\mathrm{TA})$, acetic acid $(\mathrm{AcOH})$ and benzoic acid $(\mathrm{BA})$ were also tested at $0{ }^{\circ} \mathrm{C}$ and room temperature to determine the optimum conditions with the best organocatalyst $7 \mathrm{c}$. As it can be seen from the Table 2 , the best results were obtained at $0{ }^{\circ} \mathrm{C}$ in the presence of $\mathrm{BA}$ as co-catalyst in water.

With these promising results, the substrates in the reaction were broadened with different aliphatic ketones and aromatic aldehydes in the presence of organocatalyst $7 \mathrm{c}$ under the optimum conditions (Table 3). All aldol products are known in the literature, and their structures

Table 2. Catalytic acitivity of organocatalyst $7 \mathrm{c}$ under various conditions.

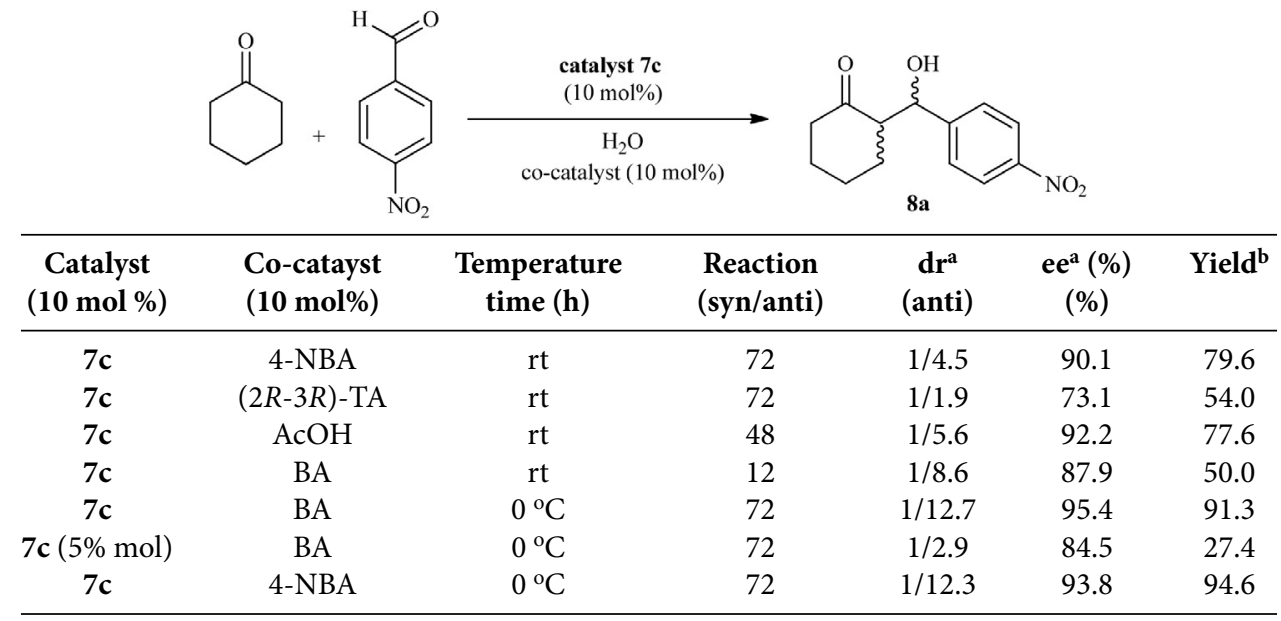

${ }^{a}$ Determined by chiral-phase HPLC analysis. ${ }^{\mathrm{b}}$ Combined yields of isolated diastereomers. 
Table 3. Aldol reactions of different aldehydes and ketones catalyzed by $7 \mathrm{c}$.

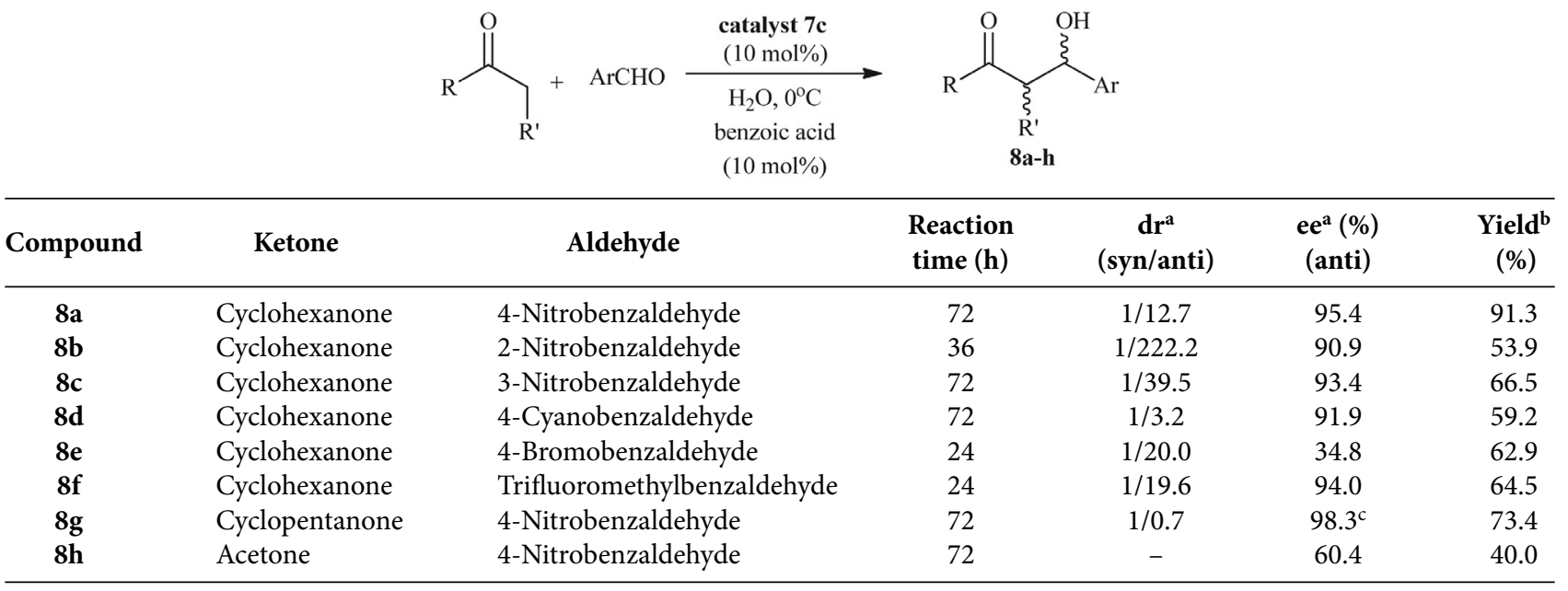

${ }^{\mathrm{a}}$ Determined by chiral-phase HPLC analysis. ${ }^{\mathrm{b}}$ Combined yields of isolated diastereomers. ${ }^{\mathrm{c}}$ syn isomer.

are in agreement with the literature data. ${ }^{39,40}$ The diastereomeric ratios and enantiomeric excesses were determined by chiral HPLC analysis of the products by using literature methods. ${ }^{41-43}$

\section{Conclusion}

In conclusion, we have designed and synthesized new di- or triamide organocatalysts derived from $(L)$-proline and successfully used in the direct asymmetric aldol reaction of aliphatic ketones and aromatic aldehydes in water. Among the catalysts investigated in this study, catalyst $7 \mathrm{c}$ gave the best diastereoselectivities (up to 99\%), enantioselectivities (up to 98\%) and yields (up to 91\%) when different aliphatic ketones and aromatic aldehydes with electron withdrawing groups were used. Furthermore, these catalysts showed their best catalytic activities in water which is also an important contribution to green chemistry requirements.

\section{Experimental}

\section{General}

All reagents were of commercial quality and reagent quality solvents were used without further purification. IR spectra were determined on a Perkin Elmer, Spectrum One FT-IR spectrometer and Bruker Tensor 27 spectrometer. NMR spectra were recorded on Bruker Avance III 500 $\mathrm{MHz}$ and Varian-INOVA $500 \mathrm{MHz}$ NMR spectrometer. Chemical shifts $\delta$ are reported in ppm with TMS as internal standart and the solvents were $\mathrm{CDCl}_{3}$ and $\mathrm{CD}_{3} \mathrm{OD}$. Column chromatography was conducted on silica gel 60 $(40-63 \mu \mathrm{M})$. TLC was carried out on aluminum sheets precoated with silica gel $60 \mathrm{~F}_{254}$ (Merck). GC-MS spectrum was recorded on Agilent 6890N-GC-System-5973 IMSD spectrometer. LC-MS (QTOF) spectra were obtained on Agilent G6530B model TOF/Q-TOF Mass Spectrometer. Optical rotations were measured with Bellingham Stanley ADP-410 Polarimeter. Chiral HPLC analyses were performed with Shimadzu HPLC (Daicel Chiralpak AD and AD-H columns) equipped with SPD-20A detector and isopropanol/hexane mixtures as the eluent. The protection of $L$-proline was carried out according to the literature procedure. ${ }^{44}$ Spectroscopic data of this compound were in accordance with its structure.

\section{General Procedure for the Synthesis of Aminobenzamid Derivatives (1a-d)}

The corresponding amines $(1.15 \mathrm{mmol})$ were added to isatoic anhydride $(1.00 \mathrm{mmol})$, dissolved in ethyl acetate and stirred at room tempearature for 5 hours (for compound $1 \mathrm{a}$ at $60{ }^{\circ} \mathrm{C}$ for 1.5 hours). The precipitates were filtered and the crude products were purified by crystallization or column chromatography.

\section{General Procedure for Amidation Reactions}

1-Hydroxy- $1 \mathrm{H}$-benzotriazole ( $\mathrm{HOBt}, 1.00 \mathrm{mmol}$ ) was added to the stirred solution of Boc-protected acid $(0.92 \mathrm{mmol})$ in dry THF. After $10 \mathrm{~min}$ stirring at $0{ }^{\circ} \mathrm{C}$ under nitrogen, dicyclohexylcarbodiimide (DCC) $(1.00$ mmol) was added. The mixture was stirred at $0{ }^{\circ} \mathrm{C}$ for $1 \mathrm{~h}$, and the amine $(1.02 \mathrm{mmol})$ was added. In the case of amino acid ester hydrochloride, to the suspension of amino acid ester hydrochloride in dry THF, triethylamine (0.5 $\mathrm{mL}$ ) was added, and stirred at room temperature for $1 \mathrm{~h}$. This solution was then added to the first mixture. The reaction mixture was then stirred at room temperature for 24 $\mathrm{h}$, and the reaction monitored by TLC. The formed precipitate was removed by filtration, and filtrate evaporated under vacuum. The residue was dissolved in $50 \mathrm{~mL}$ of ethyl acetate, and resultant solution washed successively with 
saturated aqueous solution of $\mathrm{NaHCO}_{3}(30 \mathrm{~mL} \times 3), 5 \%$ aqueous solution of $\mathrm{KHSO}_{4}(30 \mathrm{~mL} \times 3)$ and saturated aqueous solution of $\mathrm{NaCl}(30 \mathrm{~mL} \times 3)$ and finally dried with anhydrous $\mathrm{Na}_{2} \mathrm{SO}_{4}$. After filtration, the mixture was evaporated under vacuum to give the crude products $2 \mathbf{a}-$ d, 4a, 4c and 6a-c which were purified by column chromatograpy on silica gel.

General Procedure for Hydrolysis to Synthesize $5 \mathrm{a}$ and $5 \mathrm{c}$ To the solution of compounds $4 \mathrm{a}$ and $4 \mathrm{c}(1.00 \mathrm{mmol})$ in methanol $(5 \mathrm{~mL}), 2 \mathrm{~N}$ aqueous $\mathrm{NaOH}$ was added at $0{ }^{\circ} \mathrm{C}$ to adjust to $\mathrm{pH} 11$. The reaction mixture was stirred at $0{ }^{\circ} \mathrm{C}$ for $3 \mathrm{~h}$, and at room temperature for $24 \mathrm{~h}$, and then adjusted to $\mathrm{pH} 2$ with aqueous solution of $\mathrm{KHSO}_{4}$. The solution was evaporated under vacuum to remove methanol, and extracted with ethyl acetate $(30 \mathrm{~mL} \times 3)$. The combined organic layer was successively washed with brine $(20 \mathrm{~mL} \times$ 2) and dried with anhydrous $\mathrm{Na}_{2} \mathrm{SO}_{4}$. After filtration, the filtrate was evaporated to provide compounds $\mathbf{5 a}$ and $\mathbf{5 c}$ which was used without any further purification.

\section{General Procedure for Deprotection and Synthesis of $3 a-d$ and $7 \mathbf{a}-\mathbf{c}$}

To the solution of $N$-Boc protected compounds $2 \mathbf{a}-\mathbf{d}$ and $6 \mathbf{a}-\mathbf{c}(1.00 \mathrm{mmol})$ in dry DCM $(15 \mathrm{~mL})$ at $0{ }^{\circ} \mathrm{C}$, TFA $(27.00 \mathrm{mmol})$ was added dropwise over $5 \mathrm{~min}$ with stirring. The reaction mixture was stirred at $0{ }^{\circ} \mathrm{C}$ for $1 \mathrm{~h}$, and at room temperature for $2 \mathrm{~h}$, then $2 \mathrm{M} \mathrm{K}_{2} \mathrm{CO}_{3}$ was added to the reaction mixture to adjust basic $\mathrm{pH}$. The organic phase was washed with water, dried over $\mathrm{MgSO}_{4}$, filtered and evaporated to give the pure compounds $3 \mathbf{a}-\mathbf{d}$ and $7 \mathbf{a}-\mathbf{c}$.

\section{(S)-2-Amino- $\mathrm{N}$-(1-phenylethyl)benzamide (1a)}

White solid, yield $71 \%$, mp $132.4-133.2{ }^{\circ} \mathrm{C}$, (mp $\left.136-138{ }^{\circ} \mathrm{C},{ }^{37}\right),\left[\alpha{ }^{20}{ }_{\mathrm{D}}=-103.9\left(\mathrm{c}=1.02, \mathrm{CHCl}_{3}\right) ;{ }^{1} \mathrm{H}\right.$ $\operatorname{NMR}\left(\mathrm{CDCl}_{3}, 500 \mathrm{MHz}\right) \delta 1.57\left(\mathrm{~d}, J=6.9 \mathrm{~Hz}, 3 \mathrm{H}, \mathrm{CH}_{3}\right)$, 5.23-5.29 (m, 1H, $\left.\mathrm{CHCH}_{3}\right), 6.29$ (brs, $\left.1 \mathrm{H}, \mathrm{NH}\right), 5.49$ (brs, $\left.2 \mathrm{H}, \mathrm{NH}_{2}\right), 6.61-6.66(\mathrm{~m}, 2 \mathrm{H}, \mathrm{ArH}), 7.17-7.20(\mathrm{~m}, 1 \mathrm{H}$, ArH), 7.25-7.28 (m, 1H, ArH), 7.31-7.38 (m, 5H, ArH) ppm; FTIR (ATR) v 3418, 3299, 3083, 3057, 3029, 2984, 2971, 1618, 1531, 1493, 1447, $1156 \mathrm{~cm}^{-1}$; GC-MS: $m / z 240$ $\left(\mathrm{M}^{+}\right), 136\left(\mathrm{C}_{7} \mathrm{H}_{8} \mathrm{~N}_{2} \mathrm{O}\right), 120\left(\mathrm{C}_{7} \mathrm{H}_{7} \mathrm{NO}-1\right), 105\left(\mathrm{C}_{7} \mathrm{H}_{6} \mathrm{O}-1\right)$, $92\left(\mathrm{C}_{7} \mathrm{H}_{8}\right), 77\left(\mathrm{C}_{6} \mathrm{H}_{6}-1\right)$.

\section{(R)-2-Amino- $\mathrm{N}$-(1-phenylethyl)benzamide (1b)}

White solid, yield $95 \%$, mp $132.6-133.8^{\circ} \mathrm{C},[\alpha]^{20}{ }_{\mathrm{D}}=$ $+82.0\left(\mathrm{c}=1.00, \mathrm{CHCl}_{3}\right)$, [30]. FTIR (ATR) v 3417, 3298, 3084, 3057, 3028, 2984, 2927, 1618, 1531, 1493, 1447, 1346, $1156 \mathrm{~cm}^{-1}$.

\section{2-Amino- $\mathrm{N}$-butylbenzamide (1c)}

White solid, yield $95 \%$, mp 88.5-89.2 ${ }^{\circ} \mathrm{C} ;{ }^{38}{ }^{1} \mathrm{H}$ NMR $\left(\mathrm{CDCl}_{3}, 500 \mathrm{MHz}\right) \delta 0.93\left(\mathrm{td}, J=7.3,1.4 \mathrm{~Hz}, 3 \mathrm{H}, \mathrm{CH}_{3}\right)$, 1.34-1.41 (m, 2H, $\left.\mathrm{CH}_{2}\right), 1.52-1.58\left(\mathrm{~m}, 2 \mathrm{H}, \mathrm{CH}_{2}\right), 3.34-$ $3.39\left(\mathrm{~m}, 2 \mathrm{H}, \mathrm{CH}_{2}\right), 5.34$ (brs, $\left.2 \mathrm{H}, \mathrm{NH}_{2}\right), 6.20$ (brs, $1 \mathrm{H}$, $\mathrm{NH}), 6.59-6.62(\mathrm{~m}, 1 \mathrm{H}, \mathrm{ArH}), 6.65(\mathrm{~d}, J=8.2 \mathrm{~Hz}, 1 \mathrm{H}$,
ArH), 7.15-7.18 (m, 1H, ArH), $7.29(\mathrm{~d}, J=8.0 \mathrm{~Hz}, 1 \mathrm{H}$, ArH) ppm; FTIR (ATR) v 3421, 3303, 3076, 2958, 2931, $2872,1633,1531,1488,1448,1367,1155 \mathrm{~cm}^{-1}$.

\section{2-Amino- $N$-cyclohexylbenzamide (1d)}

White solid, yield $82 \%$, mp $156.9^{\circ} \mathrm{C} ; 3^{38}{ }^{1} \mathrm{H}$ NMR $\left(\mathrm{CDCl}_{3}, 500 \mathrm{MHz}\right) \delta 1.17-1.23\left(\mathrm{~m}, 1 \mathrm{H}, \mathrm{CH}_{2}\right), 1.35-1.40$ (m, $\left.4 \mathrm{H}, 2 \times \mathrm{CH}_{2}\right), 1.64-1.66\left(\mathrm{~m}, 1 \mathrm{H}, \mathrm{CH}_{2}\right), 1.76-1.79(\mathrm{~m}$, $\left.2 \mathrm{H}, \mathrm{CH}_{2}\right), 1.95-1.96\left(\mathrm{~m}, 2 \mathrm{H}, \mathrm{CH}_{2}\right), 3.86-3.93(\mathrm{~m}, 1 \mathrm{H}$, $\mathrm{C} \underline{\mathrm{HNH}}), 6.19$ (brs, $\left.2 \mathrm{H}, \mathrm{NH}_{2}\right), 6.49-6.52(\mathrm{~m}, 1 \mathrm{H}, \mathrm{ArH})$, $6.74(\mathrm{~d}, J=8.1 \mathrm{~Hz}, 1 \mathrm{H}, \mathrm{ArH}), 7.11-7.14(\mathrm{~m}, 1 \mathrm{H}, \mathrm{ArH}), 7.25$ (brs, $1 \mathrm{H}, \mathrm{NH}), 7.48(\mathrm{~d}, J=7.8 \mathrm{~Hz}, 1 \mathrm{H}, \mathrm{ArH})$ ppm; FTIR (ATR) v 3462, 3355, 3279, 3056, 2930, 2849, 1616, 1538, $1494,1463,1447,1151 \mathrm{~cm}^{-1}$.

(S)-tert-Butyl 2-(2-((S)-1-phenylethylcarbamoyl)phenylcarbamoyl)pyrrolidine-1-carboxylate (2a)

Colorless oil, yield $73.1 \%,[\alpha]^{20}{ }_{D}=-88.00(\mathrm{c}=1.00$, $\left.\mathrm{CHCl}_{3}\right) ;{ }^{1} \mathrm{H} \mathrm{NMR}\left(\mathrm{CDCl}_{3}, 500 \mathrm{MHz}\right) \delta 1.36$ and $1.53(\mathrm{~s}$, $9 \mathrm{H}, 3 \times \mathrm{CH}_{3}$, rotamers), $1.59\left(\mathrm{~d}, J=6.9 \mathrm{~Hz}, 3 \mathrm{H}, \mathrm{CH}_{3}\right)$, $1.77-1.80(\mathrm{~m}, 2 \mathrm{H}$, pro- $\gamma), 2.02-2.08(\mathrm{~m}, 1 \mathrm{H}$, pro- $\beta), 2.11-$ $2.13(\mathrm{~m}, 1 \mathrm{H}$, pro- $\beta$ ), 3.46-3.51 (m, $1 \mathrm{H}$, pro- $\delta), 3.61-3.66$ (m, $1 \mathrm{H}$, pro- $\delta$ ), $4.22-4.25$ and $4.38-4.41(\mathrm{~m}, 1 \mathrm{H}$, pro- $\alpha$, rotamers), $5.27-5.32(\mathrm{~m}, 1 \mathrm{H}, \mathrm{PhCH}), 6.55$ (brd, $J=6.7 \mathrm{~Hz}$, $1 \mathrm{H}, \mathrm{NH}), 7.05-7.11(\mathrm{~m}, 1 \mathrm{H}, \mathrm{ArH}), 7.29-7.32(\mathrm{~m}, 1 \mathrm{H}$, ArH), 7.35-7.38 (m, 4H, ArH), 7.42-7.51 (m, 2H, ArH), 8.61-8.63 (m, 1H, ArH), 11.40 and 11.43 (brs, $1 \mathrm{H}, \mathrm{NH}$, rotamers) $\mathrm{ppm} ;{ }^{13} \mathrm{C} \mathrm{NMR}\left(\mathrm{CDCl}_{3}, 125 \mathrm{MHz}\right) \delta 21.7\left(\mathrm{CH}_{3}\right)$, 23.7 (pro- $\gamma$ ), $28.2\left(3 \mathrm{xCH}_{3}\right), 31.4$ (pro- $\beta$ ), 46.6 (pro- $\delta$ ), 49.2 $(\mathrm{PhCH}), 63.7$ (pro- $\alpha), 80.1\left(\mathrm{C}_{\left.\left(\mathrm{CH}_{3}\right)_{3}\right),} 120.8\left(\mathrm{C}_{\text {aro }} \mathrm{H}\right)\right.$, $121.1\left(\mathrm{C}_{\text {aro }} \mathrm{H}\right), 122.9\left(\mathrm{C}_{\text {aro }} \mathrm{H}\right), 126.1\left(\mathrm{C}_{\text {aro }} \mathrm{H}\right), 126.4\left(\mathrm{C}_{\text {aro }} \mathrm{H}\right)$, $127.5\left(\mathrm{C}_{\text {aro }} \mathrm{H}\right), 128.7\left(\mathrm{C}_{\text {aro }} \mathrm{H}\right), 132.6\left(\mathrm{C}_{\text {aro }}\right), 139.2\left(\mathrm{C}_{\text {aro }}\right)$, $142.9\left(\mathrm{C}_{\text {aro }}\right), 154.1(\mathrm{C}=\mathrm{O}), 167.8(\mathrm{C}=\mathrm{O}), 172.3(\mathrm{C}=\mathrm{O}) \mathrm{ppm}$; LC-MS (ESI-QTOF) $m / z[\mathrm{M}+\mathrm{H}]^{+}$, found $438.2389 . \mathrm{C}_{25} \mathrm{H}-$ ${ }_{31} \mathrm{~N}_{3} \mathrm{O}_{4}$ requires 438.2393. FTIR (ATR) v 3323, 3033, 2970, $2927,1688,1580,1505,1448,1156 \mathrm{~cm}^{-1}$.

(S)-tert-Butyl 2-(2-((R)-1-phenylethylcarbamoyl)phenylcarbamoyl)pyrrolidin-1-carboxylate (2b)

Pinkish solid, yield $27 \%$, mp $54.4{ }^{\circ} \mathrm{C},[\alpha]^{20}{ }_{\mathrm{D}}=-8.0(\mathrm{c}$ $\left.=1.00, \mathrm{CHCl}_{3}\right) ;{ }^{1} \mathrm{H} \mathrm{NMR}\left(\mathrm{CDCl}_{3}, 500 \mathrm{MHz}\right) \delta 1.32$ and $1.53\left(\mathrm{~s}, 9 \mathrm{H}, 3 \times \mathrm{CH}_{3}\right.$, rotamers), 1.59 and $1.62(\mathrm{~d}, J=6.6$ $\mathrm{Hz}, 3 \mathrm{H}, \mathrm{CH}_{3}$, rotamers), $1.86-1.91$ (m, $1 \mathrm{H}$, pro- $\gamma$ ), $1.93-$ $2.02(\mathrm{~m}, 1 \mathrm{H}$, pro- $\gamma), 2.08-2.16$ and $2.21-2.29(\mathrm{~m}, 2 \mathrm{H}$, pro- $\beta$, rotamers), $3.41-3.47$ and $3.51-3.56(\mathrm{~m}, 1 \mathrm{H}$, pro- $\delta$, rotamers), $3.66-3.76(\mathrm{~m}, 1 \mathrm{H}$, pro- $\delta$ ), $4.22-4.25$ and $4.38-$ 4.40 (dd, $J=8.5,4.1 \mathrm{~Hz}, 1 \mathrm{H}$, pro- $\alpha$, rotamers), $5.27-5.33$ $(\mathrm{m}, 1 \mathrm{H}, \mathrm{PhCH}), 6.48$ and $6.64(\mathrm{brd}, J=7.5 \mathrm{~Hz}, 1 \mathrm{H}, \mathrm{NH}$, rotamers), $7.00-7.08(\mathrm{~m}, 1 \mathrm{H}, \mathrm{ArH}), 7.28-7.31(\mathrm{~m}, 1 \mathrm{H}$, ArH), 7.36-7.39 (m, 4H, ArH), 7.44-7.48 (m, 2H, ArH), 8.57-8.63 (m, 1H, ArH), 11.48 and 11.56 (brs, $1 \mathrm{H}, \mathrm{NH}$, rotamers) ppm; ${ }^{13} \mathrm{CNMR}\left(\mathrm{CDCl}_{3}, 125 \mathrm{MHz}\right) \delta 19.1\left(\mathrm{CH}_{3}\right)$, 21.6 (pro- $\gamma), 28.2\left(3 \times \mathrm{CH}_{3}\right), 31.4$ (pro- $\left.\beta\right), 46.7$ (pro- $\delta$ ), $49.1(\mathrm{Ph} \underline{\mathrm{CH}}), 62.4$ (pro- $\alpha), 80.0\left(\mathrm{C}\left(\mathrm{CH}_{3}\right)_{3}\right), 120.0\left(\mathrm{C}_{\text {aro }} \mathrm{H}\right)$, 121.0 $\left(\mathrm{C}_{\text {aro }} \mathrm{H}\right), 122.8\left(\mathrm{C}_{\text {aro }} \mathrm{H}\right), 126.3\left(\mathrm{C}_{\text {aro }} \mathrm{H}\right), 126.6\left(\mathrm{C}_{\text {aro }} \mathrm{H}\right)$, $127.5\left(\mathrm{C}_{\text {aro }} \mathrm{H}\right), 128.7\left(\mathrm{C}_{\text {aro }} \mathrm{H}\right), 132.4\left(\mathrm{C}_{\text {aro }}\right), 139.2\left(\mathrm{C}_{\text {aro }}\right)$, 
$142.7\left(\mathrm{C}_{\text {aro }}\right), 154.1(\mathrm{C}=\mathrm{O}), 167.8(\mathrm{C}=\mathrm{O}), 172.2(\mathrm{C}=\mathrm{O}) \mathrm{ppm}$; LC-MS (ESI-QTOF): $m / z[\mathrm{M}+\mathrm{H}]^{+}$, found $438.2389 . \mathrm{C}_{25} \mathrm{H}-$ ${ }_{31} \mathrm{~N}_{3} \mathrm{O}_{4}$ requires 438.2393; FTIR (ATR): $v=3313,3065$, $2974,2931,1676,1642,1585,1444,1386,1158 \mathrm{~cm}^{-1}$.

\section{(S)-tert-Butyl 2-(2-(butylcarbamoyl)phenylcarbamoyl) pyrrolidine-1-carboxylate (2c)}

Yellow oil, yield $85 \%,[\alpha]^{20}{ }_{D}=-64.0(\mathrm{c}=1.00$, $\left.\mathrm{CHCl}_{3}\right) ;{ }^{1} \mathrm{H}$ NMR $\left(\mathrm{CDCl}_{3}, 500 \mathrm{MHz}\right) \delta 0.87$ (brs, $3 \mathrm{H}$, $\left.\mathrm{CH}_{3}\right), 1.26$ and 1.41 (s, $9 \mathrm{H}, 3 \times \mathrm{CH}_{3}$, rotamers), 1.29-1.32 $\left(\mathrm{m}, 2 \mathrm{H}, \mathrm{CH}_{2}\right), 1.49-1.51\left(\mathrm{~m}, 2 \mathrm{H}, \mathrm{CH}_{2}\right), 1.79-1.81(\mathrm{~m}, 1 \mathrm{H}$, pro- $\gamma$ ), $1.86-1.90(\mathrm{~m}, 1 \mathrm{H}$, pro- $\gamma), 2.00-2.09$ and $2.16-2.20$ (m, $2 \mathrm{H}$, pro- $\beta$ ), 3.26-3.31 (m, $\left.1 \mathrm{H}, \mathrm{NCH}_{2}\right), 3.32-3.36(\mathrm{~m}$, $\left.1 \mathrm{H}, \mathrm{NCH}_{2}\right), 3.41-3.46(\mathrm{~m}, 1 \mathrm{H}$, pro- $\delta), 3.59-3.65(\mathrm{~m}, 1 \mathrm{H}$, pro- $\delta$ ), $4.13-4.16$ and $4.28-4.30$ (m, $1 \mathrm{H}$, pro- $\alpha$, rotamers), 6.68 and 6.72 (brs, $1 \mathrm{H}, \mathrm{NH}$, rotamers), $6.90-7.00(\mathrm{~m}, 1 \mathrm{H}$, ArH), 7.26-7.46 (m, 2H, ArH), 8.48 and 8.52 (brd, $1 \mathrm{H}$, $\mathrm{ArH}$, rotamers), 11.51 (brs, $1 \mathrm{H}, \mathrm{NH}) \mathrm{ppm} ;{ }^{13} \mathrm{C} \mathrm{NMR}$ $\left(\mathrm{CDCl}_{3}, 125 \mathrm{MHz}\right) \delta 13.8\left(\mathrm{CH}_{3}\right), 20.1\left(\mathrm{CH}_{2}\right), 23.7\left(\mathrm{CH}_{2}\right)$, $28.2\left(3 \times \mathrm{CH}_{3}\right), 30.5$ (pro- $\left.\gamma\right), 31.5($ pro- $\beta), 39.6\left(\mathrm{NHCH}_{2}\right)$, 46.7 (pro- $\delta), 62.4$ (pro- $\alpha), 80.0\left(\underline{\mathrm{C}}\left(\mathrm{CH}_{3}\right)_{3}\right), 120.8\left(\mathrm{C}_{\text {aro }} \mathrm{H}\right)$, $120.9\left(\mathrm{C}_{\text {aro }} \mathrm{H}\right), 122.8\left(\mathrm{C}_{\text {aro }} \mathrm{H}\right), 126.7\left(\mathrm{C}_{\text {aro }} \mathrm{H}\right), 132.2\left(\mathrm{C}_{\text {aro }}\right)$, $139.0\left(\mathrm{C}_{\text {aro }}\right), 154.1(\mathrm{C}=\mathrm{O}), 168.6(\mathrm{C}=\mathrm{O}), 172.2(\mathrm{C}=\mathrm{O}) \mathrm{ppm}$; LC-MS (ESI-QTOF) $m / z[\mathrm{M}+\mathrm{H}]^{+}$, found $390.2388 . \mathrm{C}_{21} \mathrm{H}-$ ${ }_{31} \mathrm{~N}_{3} \mathrm{O}_{4}$ requires 390.2393; FTIR (ATR) v 3351, 3067, 2957, $2931,2873,1679,1644,1585,1516,1477,1446 \mathrm{~cm}^{-1}$.

(S)-tert-Butyl 2-(2-(cyclohexylcarbamoyl)phenylcarbamoyl)pyrrolidine-1-carboxylate (2d)

Yellow solid, yield $86 \%$, mp $139.2-140.1{ }^{\circ} \mathrm{C},[\alpha]^{20}{ }_{\mathrm{D}}=$ $-76.0\left(\mathrm{c}=1.00, \mathrm{CHCl}_{3}\right) ;{ }^{1} \mathrm{H}$ NMR $\left(\mathrm{CDCl}_{3}, 500 \mathrm{MHz}\right) \delta$ 1.19-1.26 (m, $\left.3 \mathrm{H}, \mathrm{CH}_{2}\right), 1.35$ and $1.50\left(\mathrm{~s}, 9 \mathrm{H}, 3 \times \mathrm{CH}_{3}\right.$, rotamers $), 1.41-1.43\left(\mathrm{~m}, 2 \mathrm{H}, \mathrm{CH}_{2}\right), 1.64-1.67(\mathrm{~m}, 1 \mathrm{H}$, $\left.\mathrm{CH}_{2}\right), 1.74-1.76\left(\mathrm{~m}, 2 \mathrm{H}, \mathrm{CH}_{2}\right), 1.87-1.92\left(\mathrm{~m}, 1 \mathrm{H}, \mathrm{CH}_{2}\right)$, 1.96-1.98 (m, $3 \mathrm{H}, \mathrm{CH}_{2}$ ve pro- $\left.\gamma\right), 2.08-2.11(\mathrm{~m}, 1 \mathrm{H}$, pro- $\beta$ ), $2.16-2.19(\mathrm{~m}, 1 \mathrm{H}$, pro- $\beta$ ), $3.41-3.47$ and $3.51-3.56$ (m, $1 \mathrm{H}$, pro- $\delta), 3.67-3.75(\mathrm{~m}, 1 \mathrm{H}$, pro- $\delta), 3.91-3.95(\mathrm{~m}$, $1 \mathrm{H}, \mathrm{NH} \underline{\mathrm{CH}}), 4.22-4.25$ and $4.39-4.42(\mathrm{~m}, 1 \mathrm{H}$, pro- $\mathrm{\alpha}$, rotamers), 6.11 and 6.17 (brd, $J=7.3 \mathrm{~Hz}, 1 \mathrm{H}, \mathrm{NH}), 7.02-7.09$ (m, 1H, ArH), 7.39-7.47 (m, 2H, ArH), 8.61-8.63 (m, 1H, ArH), 11.47 (brs, $1 \mathrm{H}, \mathrm{NH}) \mathrm{ppm} ;{ }^{13} \mathrm{C}$ NMR $\left(\mathrm{CDCl}_{3}, 125\right.$ MHz) $\delta 23.8\left(\mathrm{CH}_{2}\right), 24.8\left(\mathrm{CH}_{2}\right), 25.5\left(\mathrm{CH}_{2}\right), 28.2\left(3 \mathrm{xCH}_{3}\right)$, $30.5\left(\mathrm{CH}_{2}\right), 31.5$ (pro- $\gamma$ ), $32.9\left(\mathrm{CH}_{2}\right), 33.0$ (pro- $\left.\beta\right), 47.7$ $(\mathrm{N}-\mathrm{CH}), 48.5$ (pro- $\delta$ ), 62.5 (pro- $\alpha$ ), $80.0\left(\underline{\mathrm{C}}\left(\mathrm{CH}_{3}\right)_{3}\right), 121.1$ $\left(\mathrm{C}_{\text {aro }} \mathrm{H}\right), 121.3\left(\mathrm{C}_{\text {aro }} \mathrm{H}\right), 122.8\left(\mathrm{C}_{\text {aro }} \mathrm{H}\right), 126.4\left(\mathrm{C}_{\text {aro }} \mathrm{H}\right), 132.4$ $\left(\mathrm{C}_{\text {aro }}\right), 139.1\left(\mathrm{C}_{\text {aro }}\right), 154.1(\mathrm{C}=\mathrm{O}), 167.7(\mathrm{C}=\mathrm{O}), 172.2$ $(\mathrm{C}=\mathrm{O}) \mathrm{ppm}$; LC-MS (ESI-QTOF) $\mathrm{m} / z[\mathrm{M}+\mathrm{H}]^{+}$, found 416.2545. $\mathrm{C}_{23} \mathrm{H}_{33} \mathrm{~N}_{3} \mathrm{O}_{4}$ requires 416.2549; FTIR (ATR) v $3326,3064,2957,2930,2873,1683,1641,1586,1446,1366$, $1160 \mathrm{~cm}^{-1}$.

(2S)-N-(2-((1S)-1-phenylethylcarbamoyl)phenyl)pyrrolidin-2-carboxamide (3a)

White solid, yield $57 \%, \mathrm{mp} 161.2-162.1^{\circ} \mathrm{C},[\alpha]^{20}{ }_{\mathrm{D}}=$ $-40.9\left(\mathrm{c}=1.27, \mathrm{CHCl}_{3}\right) ;{ }^{1} \mathrm{H}$ NMR $\left(\mathrm{CDCl}_{3}, 500 \mathrm{MHz}\right) \delta$ $1.53\left(\mathrm{~d}, J=6.9 \mathrm{~Hz}, 3 \mathrm{H}, \mathrm{CH}_{3}\right), 1.55-1.60(\mathrm{~m}, 2 \mathrm{H}$, pro- $\gamma)$,
1.85-1.91 (m, $1 \mathrm{H}$, pro- $\beta$ ), 1.99 (brs, $1 \mathrm{H}, \mathrm{NH}), 2.04-2.11$ (m, $1 \mathrm{H}$, pro- $\beta$ ), 2.74-2.79 (m, $1 \mathrm{H}$, pro- $\delta), 2.90-2.94(\mathrm{~m}$, $1 \mathrm{H}$, pro- $\delta), 3.76-3.79(\mathrm{~m}, 1 \mathrm{H}$, pro- $\alpha), 5.22-5.27(\mathrm{~m}, 1 \mathrm{H}$, $\mathrm{PhCH}), 6.33$ (brd, $J=6.5 \mathrm{~Hz}, 1 \mathrm{H}, \mathrm{NH}), 6.97-7.00(\mathrm{~m}, 1 \mathrm{H}$, ArH), 7.18-7.21 (m, 1H, ArH), 7.25-7.30 (m, 4H, ArH), 7.34-7.39 (m, 2H, ArH), 8.44-8.46 (d, J=8.2 Hz, 1H, ArH), 11.62 (brs, $1 \mathrm{H}, \mathrm{NH}) \mathrm{ppm} ;{ }^{13} \mathrm{C} \mathrm{NMR}\left(\mathrm{CDCl}_{3}, 125 \mathrm{MHz}\right) \delta$ $21.8\left(\mathrm{CH}_{3}\right), 26.1$ (pro- $\gamma$ ), 31.1 (pro- $\beta$ ), 47.2 (pro- $\delta$ ), 49.1 $(\mathrm{PhCH}), 61.5($ pro- $\alpha), 121.7\left(\mathrm{C}_{\text {aro }} \mathrm{H}\right), 122.9\left(\mathrm{C}_{\text {aro }} \mathrm{H}\right), 123.0$ $\left(\mathrm{C}_{\text {aro }} \mathrm{H}\right), 126.1\left(\mathrm{C}_{\text {aro }} \mathrm{H}\right), 126.5\left(\mathrm{C}_{\text {aro }} \mathrm{H}\right), 127.4\left(\mathrm{C}_{\text {aro }} \mathrm{H}\right), 128.7$ $\left(\mathrm{C}_{\text {aro }} \mathrm{H}\right), 132.0\left(\mathrm{C}_{\text {aro }}\right), 138.2\left(\mathrm{C}_{\text {aro }}\right), 143.0\left(\mathrm{C}_{\text {aro }}\right), 167.6$ $(\mathrm{C}=\mathrm{O}), 174.8 \quad(\mathrm{C}=\mathrm{O})$ ppm; LC-MS (ESI-QTOF) $\mathrm{m} / \mathrm{z}$ $[\mathrm{M}+\mathrm{H}]^{+}$, found 338.1866. $\mathrm{C}_{20} \mathrm{H}_{23} \mathrm{~N}_{3} \mathrm{O}_{2}$ requires 338.1869; FTIR (ATR) v 3292, 3063, 3031, 2975, 2933, 1673, 1640, $1597,1514,1447,1374 \mathrm{~cm}^{-1}$.

(2S)-N-(2-((R)-1-phenylethylcarbamoyl)phenyl)pyrrolidine-2-carboxamide (3b)

White solid, yield $77 \%$, mp $161-161.9^{\circ} \mathrm{C},[\alpha]^{20}{ }_{\mathrm{D}}=$ $-5.8\left(\mathrm{c}=2.43, \mathrm{CHCl}_{3}\right) ;{ }^{1} \mathrm{H}$ NMR $\left(\mathrm{CDCl}_{3}, 500 \mathrm{MHz}\right) \delta 1.51$ (d, $\left.J=6.9 \mathrm{~Hz}, 3 \mathrm{H}, \mathrm{CH}_{3}\right), 1.61-1.70(\mathrm{~m}, 2 \mathrm{H}$, pro- $\gamma$ ), 1.89 $1.95(\mathrm{~m}, 1 \mathrm{H}$, pro- $\beta), 2.03$ (brs, $1 \mathrm{H}, \mathrm{NH}), 2.06-2.12(\mathrm{~m}, 1 \mathrm{H}$, pro- $\beta), 2.95-3.01(\mathrm{~m}, 2 \mathrm{H}$, pro- $\delta), 3.73-3.76(\mathrm{~m}, 1 \mathrm{H}$, pro- $\alpha$ ), 5.21-5.27 (m, $1 \mathrm{H}, \mathrm{PhCH}), 6.35$ (brd, $J=7.6 \mathrm{~Hz}$, $1 \mathrm{H}, \mathrm{NH}), 6.95-6.98(\mathrm{~m}, 1 \mathrm{H}, \mathrm{ArH}), 7.19-7.23(\mathrm{~m}, 1 \mathrm{H}$, ArH), 7.27-7.36 (m, 6H, ArH), 8.43 (brd, $J=8.2 \mathrm{~Hz}, 1 \mathrm{H}$, $\mathrm{ArH}), 11.56$ (brs, $1 \mathrm{H}, \mathrm{NH}) \mathrm{ppm} ;{ }^{13} \mathrm{C} \mathrm{NMR}\left(\mathrm{CDCl}_{3}, 125\right.$ $\mathrm{MHz}) \delta 21.5\left(\mathrm{CH}_{3}\right), 26.1$ (pro- $\left.\gamma\right), 31.1$ (pro- $\left.\beta\right), 47.3$ (pro- $\delta$ ), $49.1(\mathrm{PhCH}), 61.5($ pro- $\alpha), 121.6\left(\mathrm{C}_{\mathrm{aro}} \mathrm{H}\right), 122.9$ $\left(\mathrm{C}_{\text {aro }} \mathrm{H}\right), 123.1\left(\mathrm{C}_{\text {aro }} \mathrm{H}\right), 126.3\left(\mathrm{C}_{\text {aro }} \mathrm{H}\right), 126.6\left(\mathrm{C}_{\text {aro }} \mathrm{H}\right), 127.5$ $\left(\mathrm{C}_{\text {aro }} \mathrm{H}\right), 128.7\left(\mathrm{C}_{\text {aro }} \mathrm{H}\right), 132.0\left(\mathrm{C}_{\text {aro }}\right), 138.1\left(\mathrm{C}_{\text {aro }}\right), 142.7$ $\left(\mathrm{C}_{\text {aro }}\right), 167.7(\mathrm{C}=\mathrm{O}), 174.8(\mathrm{C}=\mathrm{O}) \mathrm{ppm}$; LC-MS (ESIQTOF) $m / z[\mathrm{M}+\mathrm{H}]^{+}$, found 338.1863. $\mathrm{C}_{20} \mathrm{H}_{23} \mathrm{~N}_{3} \mathrm{O}_{2}$ requires 338.1869; FTIR (ATR) v 3332, 3283, 3059, 3027, $2969,1656,1633,1597,1429,1343 \mathrm{~cm}^{-1}$.

\section{(S)-N-(2-(Butylcarbamoyl)phenyl)pyrrolidine-2-car-} boxamide (3c)

White solid, yield $83 \%$, mp $124.4-124.9{ }^{\circ} \mathrm{C},[\alpha]^{20}{ }_{\mathrm{D}}$ $=-3.6\left(\mathrm{c}=1.65, \mathrm{CHCl}_{3}\right) ;{ }^{1} \mathrm{H} \mathrm{NMR}\left(\mathrm{CDCl}_{3}, 500 \mathrm{MHz}\right) \delta$ $0.95\left(\mathrm{t}, J=7.0 \mathrm{~Hz}, 3 \mathrm{H}, \mathrm{CH}_{3}\right), 1.37-1.44\left(\mathrm{~m}, 2 \mathrm{H}, \mathrm{CH}_{2}\right)$, 1.56-1.62 (m, $\left.2 \mathrm{H}, \mathrm{CH}_{2}\right), 1.68-1.78(\mathrm{~m}, 2 \mathrm{H}$, pro- $\gamma), 1.98-$ $2.04(\mathrm{~m}, 1 \mathrm{H}$, pro- $\beta$ ), $2.14-2.20(\mathrm{~m}, 1 \mathrm{H}$, pro- $\beta$ ), 2.22 (brs, $1 \mathrm{H}, \mathrm{NH}), 3.02-3.11(\mathrm{~m}, 2 \mathrm{H}$, pro- $\delta), 3.35-3.42(\mathrm{~m}, 1 \mathrm{H}$, $\left.\mathrm{NCH}_{2}\right), 3.44-3.51\left(\mathrm{~m}, 1 \mathrm{H}, \mathrm{NCH}_{2}\right), 3.84-3.87(\mathrm{~m}, 1 \mathrm{H}$, pro- $\alpha$ ), 6.38 (brs, $1 \mathrm{H}, \mathrm{NH}), 7.02-7.05(\mathrm{~m}, 1 \mathrm{H}, \operatorname{ArH})$, $7.40-7.43(\mathrm{~m}, 2 \mathrm{H}, \mathrm{ArH}), 8.53(\mathrm{~d}, J=8.5 \mathrm{~Hz}, 1 \mathrm{H}, \mathrm{ArH})$, 11.74 (brs, $1 \mathrm{H}, \mathrm{NH}) \mathrm{ppm} ;{ }^{13} \mathrm{C} \mathrm{NMR}\left(\mathrm{CDCl}_{3}, 125 \mathrm{MHz}\right) \delta$ $13.8\left(\mathrm{CH}_{3}\right), 20.1\left(\mathrm{CH}_{2}\right), 26.1\left(\mathrm{CH}_{2}\right), 31.1$ (pro- $\left.\gamma\right), 31.6$ (pro- $\beta$ ), $39.7\left(\mathrm{NHCH}_{2}\right), 47.3$ (pro- $\delta$ ), 61.6 (pro- $\alpha$ ), 121.5 $\left(\mathrm{C}_{\text {aro }} \mathrm{H}\right), 122.9\left(\mathrm{C}_{\text {aro }} \mathrm{H}\right), 123.1\left(\mathrm{C}_{\text {aro }} \mathrm{H}\right), 126.6\left(\mathrm{C}_{\text {aro }} \mathrm{H}\right)$, $131.8\left(\mathrm{C}_{\text {aro }}\right), 138.1\left(\mathrm{C}_{\text {aro }}\right), 168.5(\mathrm{C}=\mathrm{O}), 174.9(\mathrm{C}=\mathrm{O}) \mathrm{ppm}$; LC-MS (ESI-QTOF) $m / z[\mathrm{M}+\mathrm{H}]^{+}$, found 290.1868 . $\mathrm{C}_{16} \mathrm{H}_{23} \mathrm{~N}_{3} \mathrm{O}_{2}$ requires 290.1869; FTIR (ATR) v 3353, 3162, $3086,3063,2960,2930,2872,1665,1632,1595,1577$, $1441,1321 \mathrm{~cm}^{-1}$. 
(S)-N-(2-(Cyclohexylcarbamoyl)phenyl)pyrrolidine-2 -carboxamide (3d)

White solid, yield $83 \%$, mp $189.6-190.3^{\circ} \mathrm{C} ;[\alpha]^{20}{ }_{\mathrm{D}}=$ $-10.0\left(\mathrm{c}=0.82, \mathrm{CHCl}_{3}\right) ;{ }^{1} \mathrm{H}$ NMR $\left(\mathrm{CDCl}_{3}, 500 \mathrm{MHz}\right) \delta$ 1.18-1.28 (m, 3H, $\left.\mathrm{CH}_{2}\right), 1.38-1.45\left(\mathrm{~m}, 2 \mathrm{H}, \mathrm{CH}_{2}\right), 1.65-$ $1.67\left(\mathrm{~m}, 1 \mathrm{H}, \mathrm{CH}_{2}\right), 1.71-1.78\left(\mathrm{~m}, 4 \mathrm{H}, 2 \times \mathrm{CH}_{2}\right), 2.00-2.05$ ( $\mathrm{m}, 3 \mathrm{H}, 2 \times$ pro- $\gamma$ ve pro- $\beta$ ), 2.08 (brs, $1 \mathrm{H}, \mathrm{NH}), 2.14-2.22$ (m, $1 \mathrm{H}$, pro- $\beta$ ), $3.01-3.12(\mathrm{~m}, 2 \mathrm{H}$, pro- $\delta), 3.85-3.88(\mathrm{~m}$, $1 \mathrm{H}, \mathrm{NCH}), 3.93-3.99$ (m, $1 \mathrm{H}$, pro- $\alpha), 6.05$ (brs, $1 \mathrm{H}, \mathrm{NH}$ ), 7.03-7.06 (m, 1H, ArH), 7.41 (d, J= 8.2 Hz, 2H, ArH), 8.54 $(\mathrm{d}, J=8.1 \mathrm{~Hz}, 1 \mathrm{H}, \mathrm{ArH}), 11.71(\mathrm{brs}, 1 \mathrm{H}, \mathrm{NH}) \mathrm{ppm} ;{ }^{13} \mathrm{C}$ NMR $\left(\mathrm{CDCl}_{3}, 125 \mathrm{MHz}\right) \delta 24.8\left(2 \times \mathrm{CH}_{2}\right), 25.5\left(\mathrm{CH}_{2}\right)$, $26.2\left(\mathrm{CH}_{2}\right), 31.1$ (pro- $\gamma$ ), $33.0\left(\mathrm{CH}_{2}\right), 33.1$ (pro- $\left.\beta\right), 47.4$ $(\mathrm{NCH}), 48.5$ (pro- $\delta$ ), 61.6 (pro- $\alpha), 121.5\left(\mathrm{C}_{\mathrm{aro}} \mathrm{H}\right), 122.9$ $\left(\mathrm{C}_{\text {aro }} \mathrm{H}\right), 123.4\left(\mathrm{C}_{\mathrm{aro}} \mathrm{H}\right), 126.6\left(\mathrm{C}_{\mathrm{aro}} \mathrm{H}\right), 131.8\left(\mathrm{C}_{\mathrm{aro}}\right), 138.1$ $\left(\mathrm{C}_{\text {aro }}\right), 167.7(\mathrm{C}=\mathrm{O}), 174.9(\mathrm{C}=\mathrm{O})$ ppm; LC-MS (ESIQTOF) $m / z[\mathrm{M}+\mathrm{H}]^{+}$, found $316.2020 . \mathrm{C}_{18} \mathrm{H}_{25} \mathrm{~N}_{3} \mathrm{O}_{2}$ requires 316.2025; FTIR (ATR) v 3462, 3355, 3279, 3056, $2930,2849,1616,1588,1538,1463,1447,1371,1187 \mathrm{~cm}^{-1}$.

(S)-tert-Butyl 2-((S)-1-(methoxycarbonyl)-2-phenylethylcarbamoyl)pyrrolidine-1-carboxylate (4a)

Yellow oil, yield $73 \%,[\alpha]^{20}{ }_{D}=-38.7(c=1.24$, $\left.\mathrm{CHCl}_{3}\right) ;{ }^{45}{ }^{1} \mathrm{H}$ NMR $\left(\mathrm{CDCl}_{3}, 500 \mathrm{MHz}\right) \delta 1.42(\mathrm{bs}, 9 \mathrm{H}$, $\left.\mathrm{C}\left(\mathrm{CH}_{3}\right)_{3}\right), 1.77-1.86(\mathrm{~m}, 2 \mathrm{H}$, pro- $\gamma), 1.88-2.06(\mathrm{~m}, 2 \mathrm{H}$, pro- $\beta$ ), $2.90-3.03\left(\mathrm{~m}, 1 \mathrm{H}, \mathrm{PhCH}_{2}\right), 3.19$ (dd, $J=14.0,5.5$ $\mathrm{Hz}, 1 \mathrm{H}, \mathrm{PhCH}_{2}$ ), 3.29-3.39 (m, $2 \mathrm{H}$, pro- $\delta$ ), 3.72 (s, $3 \mathrm{H}$, $\left.\mathrm{OCH}_{3}\right), 4.19-4.29(\mathrm{~m}, 1 \mathrm{H}$, pro- $\alpha), 4.85$ (bs, $1 \mathrm{H}$, Phe- $\left.\mathrm{CH}\right)$, 7.09 (bd, $J=7.0 \mathrm{~Hz}, 2 \mathrm{H}, \mathrm{ArH}), 7.22-7.25$ (m, 3H, ArH) ppm; ${ }^{13} \mathrm{C}$ NMR $\left(\mathrm{CD}_{3} \mathrm{OD}, 125 \mathrm{MHz}\right) \delta 24.3$ (pro- $\gamma$ ), $28.5(3$ $\left.\times \mathrm{CH}_{3}\right), 32.2($ pro- $\beta), 38.1\left(\mathrm{PhCH}_{2}\right), 47.8$ (pro- $\left.\delta\right), 52.7$ (Phe-CH), $55.1\left(\mathrm{OCH}_{3}\right), 61.6$ (pro- $\left.\alpha\right), 81.5\left(\mathrm{OC}\left(\mathrm{CH}_{3}\right)_{3}\right)$, $127.9\left(\mathrm{C}_{\text {aro }} \mathrm{H}\right), 129.5\left(\mathrm{C}_{\text {aro }} \mathrm{H}\right), 130.1\left(\mathrm{C}_{\text {aro }} \mathrm{H}\right), 138.3\left(\mathrm{C}_{\text {aro }}\right)$, $173.3(\mathrm{C}=\mathrm{O}), 175.1(\mathrm{C}=\mathrm{O}), 175.8(\mathrm{C}=\mathrm{O}) \mathrm{ppm}$; FTIR (ATR) $v 3277,3079,3028,2976,2877,1738,1689,1660,1552$, $1445,1389,1365,1210 \mathrm{~cm}^{-1}$.

(S)-tert-Butyl 2-(((S)-3-hydroxy-1-methoxy-1-oxopropane-2-yl)carbamoyl)-pyrrolidine-1-carboxylate (4c)

Colorless oil, yield $94 \%,[\alpha]^{20}{ }_{D}=+88.0(c=1.00$, $\left.\mathrm{CH}_{3} \mathrm{OH}\right) ;{ }^{46}{ }^{1} \mathrm{H}$ NMR $\left(\mathrm{CD}_{3} \mathrm{OD}, 500 \mathrm{MHz}\right) \delta 1.45$ and 1.48 (s, $9 \mathrm{H}, 3 \times \mathrm{CH}_{3}$, rotamers), $1.88-1.92(\mathrm{~m}, 1 \mathrm{H}$, pro- $\gamma$ ), $1.94-1.98(\mathrm{~m}, 1 \mathrm{H}$, pro- $\gamma), 2.00-2.05(\mathrm{~m}, 1 \mathrm{H}$, pro- $\beta), 2.12-$ $2.29(\mathrm{~m}, 1 \mathrm{H}$, pro- $\beta$ ), 3.33 (brs, $1 \mathrm{H}, \mathrm{OH}), 3.40-3.45(\mathrm{~m}, 1 \mathrm{H}$, pro- $\delta), 3.51-3.55(\mathrm{~m}, 1 \mathrm{H}$, pro- $\delta), 3.74\left(\mathrm{~s}, 3 \mathrm{H}, \mathrm{OCH}_{3}\right)$, 3.79-3.85 (m, $\left.1 \mathrm{H}, \mathrm{CH}_{2} \mathrm{OH}\right), 3.91-3.96\left(\mathrm{~m}, 1 \mathrm{H}, \mathrm{CH}_{2} \mathrm{OH}\right)$, 4.27-4.30 (m, $1 \mathrm{H}$, pro- $\alpha$ ), 4.53-4.55 (m, $\left.1 \mathrm{H}, \mathrm{CHCH}_{2} \mathrm{OH}\right)$ ppm; FTIR (ATR) v 3293, 3082, 2975, 2881, 1743, 1662, $1533,1470,1454,1205,1160 \mathrm{~cm}^{-1}$.

(S)-2-((S)-tert-Butyl 2-carbamoylpyrrolidine-1-carboxyloyl)-3-phenyl-propanoic acid (5a)

White solid, yield $96 \%$, mp $143-144{ }^{\circ} \mathrm{C}(\mathrm{mp} \mathrm{145-}$ $\left.147^{\circ} \mathrm{C}\right)^{45,47} ;[\alpha]^{20}{ }_{\mathrm{D}}=-43.1\left(\mathrm{c}=1.3, \mathrm{CHCl}_{3}\right) .{ }^{1} \mathrm{H}$ NMR $\left(\mathrm{CDCl}_{3}, 500 \mathrm{MHz}\right) \delta 1.38\left(\mathrm{~s}, 9 \mathrm{H}, \mathrm{C}\left(\mathrm{CH}_{3}\right)_{3}\right), 1.74-1.86(\mathrm{~m}$, $3 \mathrm{H}$, pro- $\gamma$ and pro- $\beta$ ), $2.06-2.09(\mathrm{~m}, 1 \mathrm{H}$, pro- $\beta$ ), 3.05-3.07 (m, $1 \mathrm{H}, \mathrm{CH}_{2} \mathrm{Ph}$ ), 3.27-3.37 (m, 3H, $\mathrm{CH}_{2} \mathrm{Ph}$ and pro- $\delta$ ), 4.25-4.29 (m, 1H, pro- $\alpha), 4.87$ (bs, $1 \mathrm{H}$, Phe-CH), 7.14$7.16(\mathrm{~m}, 2 \mathrm{H}, \mathrm{NH}$ and $\mathrm{ArH}), 7.20-7.27(\mathrm{~m}, 4 \mathrm{H}, \mathrm{ArH}) \mathrm{ppm}$; FTIR (ATR) v 3425, 3314, 3060, 3028, 2977, 2930, 2881, $1735,1660,1651,1526,1392,1367,1243,1160 \mathrm{~cm}^{-1}$.

(S)-2-((S)-1-(tert-Butoxycarbonyl)pyrrolidine-2-carboxamido)-3-hydoxypropanoic Acid (5c)

White solid, yield $67 \%$, mp $139.4-140.1{ }^{\circ} \mathrm{C} ;[\alpha]^{20}{ }_{\mathrm{D}}=$ $-106.0\left(\mathrm{c}=1.00, \mathrm{CH}_{3} \mathrm{OH}\right) ;{ }^{1} \mathrm{H} \mathrm{NMR}\left(\mathrm{CDCl}_{3}, 500 \mathrm{MHz}\right) \delta$ $1.30\left(\mathrm{~s}, 9 \mathrm{H}, 3 \mathrm{xCH}_{3}\right), 1.66-1.75(\mathrm{~m}, 2 \mathrm{H}$, pro- $\gamma), 1.90$ (brs, $2 \mathrm{H}$, pro- $\beta$ ), $3.24-3.37(\mathrm{~m}, 2 \mathrm{H}$, pro- $\delta$ ), 3.71 (brs, $1 \mathrm{H}$, $\mathrm{CH}_{2} \mathrm{OH}$ ), 3.88 (brs, $\left.1 \mathrm{H}, \mathrm{CH}_{2} \mathrm{OH}\right), 4.17-4.23(\mathrm{~m}, 2 \mathrm{H}$, pro- $\alpha$ and $\mathrm{CHCH}_{2} \mathrm{OH}$ ), 7.12 (brs, $1 \mathrm{H}, \mathrm{OH}$ ), 7.53 and 7.69 (brs, $1 \mathrm{H}, \mathrm{NH}$, rotamers) $\mathrm{ppm} ;{ }^{13} \mathrm{C} \mathrm{NMR}\left(\mathrm{CDCl}_{3}, 125\right.$ $\mathrm{MHz}) \delta 24.3$ (pro- $\gamma), 28.4\left(3 \times \mathrm{CH}_{3}\right), 30.8($ pro- $\beta), 47.3$ (pro- $\delta$ ), 56.4 (pro- $\alpha), 60.2\left(\mathrm{CHCH}_{2} \mathrm{OH}\right), 62.3\left(\mathrm{CH}_{2} \mathrm{OH}\right)$, $80.4\left(\mathrm{C}\left(\mathrm{CH}_{3}\right)_{3}\right), 155.2(\mathrm{C}=\mathrm{O}), 173.0(\mathrm{C}=\mathrm{O}), 177.8(\mathrm{C}=\mathrm{O})$ ppm; LC-MS (ESI-QTOF) $\mathrm{m} / z$ [M+Na $]^{+}$, found 325.1368 . $\mathrm{C}_{13} \mathrm{H}_{22} \mathrm{~N}_{2} \mathrm{O}_{6}$ requires 325.1376; FTIR (ATR) v 3487, 3275, $3079,2974,2932,1733,1654,1540,1479,1457,1281$, $1163 \mathrm{~cm}^{-1}$.

(S)-tert-Butyl 2-(((S)-1-(((S)-3-hydroxy-1-methoxy-1oxopropane-2-yl-amino)-1-oxo-3-phenylpropane-2-yl) carbamoyl)pyrrolidine-1-carboxylate (6a)

Colorless oil, yield $68 \%,[\alpha]^{20}{ }_{D}=+26.0(c=1.00$, $\left.\mathrm{CH}_{3} \mathrm{OH}\right) ;{ }^{48}{ }^{1} \mathrm{H}$ NMR $\left(\mathrm{CD}_{3} \mathrm{OD}, 500 \mathrm{MHz}\right) \delta 1.29$ and 1.46 (s, $9 \mathrm{H}, 3 \times \mathrm{CH}_{3}$, rotamers), $1.73-1.80(\mathrm{~m}, 3 \mathrm{H}, 2 \times$ pro- $\gamma$ and pro- $\beta), 2.06-2.16(\mathrm{~m}, 1 \mathrm{H}$, pro- $\beta), 2.91-3.04(\mathrm{~m}, 1 \mathrm{H}$, $\left.\mathrm{PhCH}_{2}\right), 3.19-3.21\left(\mathrm{~m}, 1 \mathrm{H}, \mathrm{PhCH}_{2}\right), 3.34-3.38(\mathrm{~m}, 1 \mathrm{H}$, pro- $\delta$ ), 3.41 (brs, $1 \mathrm{H}$, pro- $\delta$ ), $3.73\left(\mathrm{~s}, 3 \mathrm{H}, \mathrm{OCH}_{3}\right), 3.77-$ $3.81\left(\mathrm{~m}, 1 \mathrm{H}, \mathrm{CH}_{2} \mathrm{OH}\right), 3.88-3.89\left(\mathrm{~m}, 1 \mathrm{H}, \mathrm{CH}_{2} \mathrm{OH}\right), 4.16$ (brs, $1 \mathrm{H}$, pro- $\alpha$ ), 4.52 (brs, $1 \mathrm{H}$, Phe- $\mathrm{CH}$ ), 4.73 (brs, $1 \mathrm{H}$, $\left.\mathrm{CHCH}_{2} \mathrm{OH}\right), 7.19-7.22(\mathrm{~m}, 1 \mathrm{H}, \mathrm{ArH}), 7.26-7.29(\mathrm{~m}, 4 \mathrm{H}$, ArH) ppm; LC-MS (ESI-QTOF) $\mathrm{m} / z[\mathrm{M}+\mathrm{H}]^{+}$, found 464.2397. $\mathrm{C}_{23} \mathrm{H}_{33} \mathrm{~N}_{3} \mathrm{O}_{7}$ requires 464.2392; FTIR (ATR) v 3294, 3065, 3004, 2971, 2953, 2882, 1741, 1650, 1523, 1454, $1523,1392,1215,1160 \mathrm{~cm}^{-1}$.

(S)-tert-Butyl 2-(((S)-1-(2-(butylcarbamoyl)phenylamino)-1-oxo-3-phenylpropane-2-yl)-carbamoylpyrrolidine-1-carboxylate $(\mathbf{6 b})$

White solid, yield $52 \%, \mathrm{mp} 166.2-166.7^{\circ} \mathrm{C} ;[\mathrm{\alpha}]^{20}{ }_{\mathrm{D}}=$ $+32.0\left(\mathrm{c}=1.00, \mathrm{CH}_{3} \mathrm{OH}\right) ;{ }^{1} \mathrm{H}$ NMR $\left(\mathrm{CDCl}_{3}, 500 \mathrm{MHz}\right) \delta$ $0.89\left(\mathrm{t}, J=6.9 \mathrm{~Hz}, 3 \mathrm{H}, \mathrm{CH}_{3}\right), 1.30-1.37\left(\mathrm{~m}, 12 \mathrm{H}, 3 \times \mathrm{CH}_{3}\right.$ ve $\left.3 x b u t y l-\mathrm{CH}_{2}\right), 1.48-1.54\left(\mathrm{~m}, 2 \mathrm{H}, 1 \times\right.$ butyl $-\mathrm{CH}_{2}$ ve $1 \times$ pro- $\beta$ ), $1.67-1.77$ (m, $3 \mathrm{H}$, pro- $\gamma$ ve pro- $\beta$ ), 3.01 (brs, $1 \mathrm{H}$, $\left.\mathrm{PhCH}_{2}\right), 3.19-3.32\left(\mathrm{~m}, 5 \mathrm{H}, \mathrm{PhCH}_{2}, 2 \times\right.$ pro- $\delta, 2 \times$ $\mathrm{NHCH}_{2}$ ), 4.15 and 4.42 (brs, $1 \mathrm{H}$, pro- $\alpha$, rotamers), $4.76-$ $4.80(\mathrm{~m}, 1 \mathrm{H}$, Phe- $\mathrm{CH}$ ), 6.13 (brs, $1 \mathrm{H}, \mathrm{NH}), 6.98-7.01$ (m, 1H, ArH), 7.08-7.17 (m, 5H, ArH), 7.33-7.38 (m, 2H, ArH), 8.45 (d, $J=8.4 \mathrm{~Hz}, 1 \mathrm{H}, \mathrm{ArH}), 11.36$ (brs, $1 \mathrm{H}, \mathrm{NH}$ ) ppm; ${ }^{13} \mathrm{C}$ NMR $\left(\mathrm{CDCl}_{3}, 125 \mathrm{MHz}\right) \delta 12.7\left(\mathrm{CH}_{3}\right), 19.1$ $\left(\mathrm{CH}_{2}\right), 23.5\left(\mathrm{CH}_{2}\right), 27.3\left(3 \mathrm{xCH}_{3}\right), 30.4$ (pro- $\left.\gamma\right), 37.2$

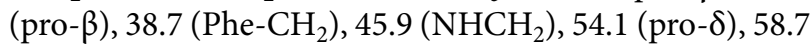

Keskin et al.: Synthesis of New Di- and Triamides ... 
(Phe-CH), 60.0 (pro- $\alpha), 79.3\left(\mathrm{C}\left(\mathrm{CH}_{3}\right)_{3}\right), 120.4\left(\mathrm{C}_{\text {aro }} \mathrm{H}\right)$, $122.0\left(\mathrm{C}_{\text {aro }} \mathrm{H}\right), 125.4\left(\mathrm{C}_{\text {aro }} \mathrm{H}\right), 125.5\left(\mathrm{C}_{\text {aro }} \mathrm{H}\right), 125.7\left(\mathrm{C}_{\mathrm{aro}} \mathrm{H}\right)$, $127.3\left(\mathrm{C}_{\text {aro }} \mathrm{H}\right), 128.3\left(\mathrm{C}_{\text {aro }} \mathrm{H}\right), 131.2\left(\mathrm{C}_{\text {aro }}\right), 135.6\left(\mathrm{C}_{\text {aro }}\right)$, $137.7\left(\mathrm{C}_{\text {aro }}\right), 154.8(\mathrm{C}=\mathrm{O}), 167.5(\mathrm{C}=\mathrm{O}), 168.7(\mathrm{C}=\mathrm{O})$, $171.1(\mathrm{C}=\mathrm{O}) \mathrm{ppm}$; LC-MS (ESI-QTOF) $\mathrm{m} / z[\mathrm{M}+\mathrm{H}]^{+}$, found 537.3071. $\mathrm{C}_{30} \mathrm{H}_{40} \mathrm{~N}_{4} \mathrm{O}_{5}$ requires 537.3071; FTIR (ATR) v 3314, 3059, 2928, 2850, 1682, 1625, 1536, 1443, $1393,1240,1163 \mathrm{~cm}^{-1}$.

(S)-tert-Butyl 2-(((S)-3-hydroxy-1- $(((S)$-1-methoxy-1oxo-3-phenylpropane-2-yl)-amino-1-oxopropane-2-yl) carbamoyl)pyrrolidine-1-carboxylate (6c)

Colorless oil, yield $62 \%,[a]^{20}{ }_{D}=-28.0(c=1.00$, $\left.\mathrm{CH}_{3} \mathrm{OH}\right) ;{ }^{1} \mathrm{H}$ NMR $\left(\mathrm{CDCl}_{3}, 500 \mathrm{MHz}\right) \delta 1.37(\mathrm{~s}, 9 \mathrm{H}$, $3 \mathrm{xCH}_{3}$ ), 1.76-1.83 (m, $2 \mathrm{H}$, pro- $\gamma$ ), 2.02 (brs, $2 \mathrm{H}$, pro- $\beta$ ), 2.95-3.00 (m, 1H, $\left.\mathrm{PhCH}_{2}\right), 3.06-3.10\left(\mathrm{~m}, 1 \mathrm{H}, \mathrm{PhCH}_{2}\right), 3.32$ (brs, $1 \mathrm{H}, \mathrm{OH}$ ), $3.36-3.43(\mathrm{~m}, 2 \mathrm{H}$, pro- $\delta$ ), 3.61 and 3.68 (s, $3 \mathrm{H}, \mathrm{OCH}_{3}$, rotamers), 3.83-3.86 (m, $\left.2 \mathrm{H}, \mathrm{CH}_{2} \mathrm{OH}\right), 4.11-$ $4.18(\mathrm{~m}, 1 \mathrm{H}$, pro- $\alpha$ ), $4.37-4.40$ (m, $1 \mathrm{H}$, Phe- $\mathrm{CH}), 4.69-4.73$ (m, $\left.1 \mathrm{H}, \mathrm{CHCH}_{2} \mathrm{OH}\right), 7.06-7.08$ (m, $\left.2 \mathrm{H}, \mathrm{ArH}\right), 7.12-7.15$ (m, 1H, ArH), 7.18-7.22 (m, 2H, ArH), 7.28 (brd, $J=6.0$ $\mathrm{Hz}, 1 \mathrm{H}, \mathrm{NH}) \mathrm{ppm} ;{ }^{13} \mathrm{C} \mathrm{NMR}\left(\mathrm{CDCl}_{3}, 125 \mathrm{MHz}\right) \delta 23.5$ (pro- $\gamma$ ), $27.3\left(3 \times \mathrm{CH}_{3}\right), 30.0$ (pro- $\left.\beta\right), 36.6\left(\mathrm{PhCH}_{2}\right), 46.2$ (pro- $\delta$ ), $51.5\left(\mathrm{OCH}_{3}\right), 52.6$ (pro- $\left.\alpha\right), 53.6\left(\mathrm{CHCH}_{2} \mathrm{OH}\right), 59.3$

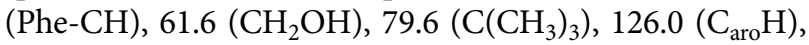
$127.5\left(\mathrm{C}_{\text {aro }} \mathrm{H}\right), 128.1\left(\mathrm{C}_{\text {aro }} \mathrm{H}\right), 135.0\left(\mathrm{C}_{\text {aro }}\right), 154.5(\mathrm{C}=\mathrm{O})$, $169.8(\mathrm{C}=\mathrm{O}), 170.8(\mathrm{C}=\mathrm{O}), 171.9(\mathrm{C}=\mathrm{O}) \mathrm{ppm}$; LC-MS (ESIQTOF) $m / z[\mathrm{M}+\mathrm{H}]^{+}$, found 464.2392. $\mathrm{C}_{23} \mathrm{H}_{33} \mathrm{~N}_{3} \mathrm{O}_{7}$ requires 464.2397; FTIR (ATR) v 3303, 3079, 2954, 2930, 1737, 1661, $1655,1535,1437,1206,1161 \mathrm{~cm}^{-1}$.

(S)-Methyl 3-hydroxy-2-((S)-3-phenyl-2-((S)-pyrrolidine-2-carboxamido)propanamidopropanoate (7a)

White solid, yield $85 \%$, mp $52^{\circ} \mathrm{C} ;[\alpha]^{20}{ }_{\mathrm{D}}=+120.0(\mathrm{c}$ $\left.=1.00, \mathrm{CH}_{3} \mathrm{OH}\right) ;{ }^{1} \mathrm{H} \mathrm{NMR}\left(\mathrm{CDCl}_{3}, 500 \mathrm{MHz}\right) \delta 1.42-1.48$ ( $\mathrm{m}, 1 \mathrm{H}$, pro- $\gamma$ ), 1.57-1.64 ( $\mathrm{m}, 2 \mathrm{H}$, pro- $\gamma$ and pro- $\beta$ ), $1.97-$ $2.04\left(\mathrm{~m}, 1 \mathrm{H}\right.$, pro- $\beta$ ), $2.73-2.77\left(\mathrm{~m}, 1 \mathrm{H}, \mathrm{PhCH}_{2}\right), 2.90-2.95$ $\left(\mathrm{m}, 1 \mathrm{H}, \mathrm{PhCH}_{2}\right), 2.96-3.01(\mathrm{~m}, 1 \mathrm{H}$, pro- $\delta), 3.22(\mathrm{dd}, J=5.5$, $14.0 \mathrm{~Hz}, 1 \mathrm{H}$, pro- $\delta$ ), 3.58 (brs, $2 \mathrm{H}$, pro- $\alpha$ and $\mathrm{OH}$ ), 3.73 (s, $\left.3 \mathrm{H}, \mathrm{OCH}_{3}\right), 3.83-3.93\left(\mathrm{~m}, 2 \mathrm{H}, \mathrm{CH}_{2} \mathrm{OH}\right), 4.58-4.61(\mathrm{~m}$, $1 \mathrm{H}$, Phe- $\mathrm{CH}$ ), 4.70 (brs, $\left.1 \mathrm{H}, \mathrm{CHCH}_{2} \mathrm{OH}\right), 7.18-7.25$ (m, $5 \mathrm{H}, \mathrm{ArH}), 7.61(\mathrm{~d}, J=7.5 \mathrm{~Hz}, 1 \mathrm{H}, \mathrm{NH}), 8.30$ (brs, $1 \mathrm{H}, \mathrm{NH})$ ppm; ${ }^{13} \mathrm{C} \mathrm{NMR}\left(\mathrm{CDCl}_{3}, 125 \mathrm{MHz}\right) \delta 25.8$ (pro- $\left.\gamma\right), 30.5$ (pro- $\beta$ ), $37.9\left(\mathrm{PhCH}_{2}\right), 47.0$ (pro- $\delta$ ), $52.6\left(\mathrm{OCH}_{3}\right), 54.1$ (pro- $\alpha$ ), $54.9\left(\mathrm{CHCH}_{2} \mathrm{OH}\right), 60.1$ (Phe- $\left.\mathrm{CH}\right), 62.4\left(\mathrm{CH}_{2} \mathrm{OH}\right)$, $126.9\left(\mathrm{C}_{\text {aro }} \mathrm{H}\right), 128.4\left(\mathrm{C}_{\text {aro }} \mathrm{H}\right), 129.3\left(\mathrm{C}_{\text {aro }} \mathrm{H}\right), 136.5\left(\mathrm{C}_{\text {aro }}\right)$, $170.8(\mathrm{C}=\mathrm{O}), 171.3(\mathrm{C}=\mathrm{O}), 175.8(\mathrm{C}=\mathrm{O})$ ppm; LC-MS (ESI-QTOF) $m / z[\mathrm{M}+\mathrm{H}]^{+}$, found 364.1869. $\mathrm{C}_{18} \mathrm{H}_{25} \mathrm{~N}_{3} \mathrm{O}_{5}$ requires 364.1872; FTIR (ATR) v 3272, 3100, 2963, 1660, $1559,1442,1299,1189,1132 \mathrm{~cm}^{-1}$.

(S)-N-((S)-1-(2-(Butylcarbamoyl) phenylamino-1-oxo -3-phenylpropane-2-yl)pyrrolidine-2-carboxamide (7b)

White solid, yield $72 \%$, mp $117-118{ }^{\circ} \mathrm{C} ;[\alpha]^{20}{ }_{\mathrm{D}}=$ $-96.0\left(\mathrm{c}=1.00, \mathrm{CHCl}_{3}\right) ;{ }^{1} \mathrm{H}$ NMR $\left(\mathrm{CDCl}_{3}, 500 \mathrm{MHz}\right) \delta$ $0.95\left(\mathrm{t}, J=7.5 \mathrm{~Hz}, 3 \mathrm{H}, \mathrm{CH}_{3}\right), 1.35-1.42\left(\mathrm{~m}, 2 \mathrm{H}\right.$, butyl $\left.-\mathrm{CH}_{2}\right)$,
1.44-1.50 (m, $1 \mathrm{H}$, pro- $\gamma), 1.53-1.59\left(\mathrm{~m}, 2 \mathrm{H}\right.$, butyl $\left.-\mathrm{CH}_{2}\right)$, 1.61-1.67 (m, $2 \mathrm{H}$, pro- $\gamma$ ve pro- $\beta), 2.02-2.06(\mathrm{~m}, 1 \mathrm{H}$, pro- $\beta$ ), $2.80-2.84\left(\mathrm{~m}, 1 \mathrm{H}, \mathrm{PhCH}_{2}\right), 2.90-2.95(\mathrm{~m}, 1 \mathrm{H}$, $\mathrm{PhCH}_{2}$ ), 3.07-3.11 (m, $1 \mathrm{H}$, pro- $\delta$ ), 3.28-3.37 (m, $3 \mathrm{H}, 2 \times$ butyl- $\mathrm{CH}_{2}$, pro- $\delta$ ), $3.79-3.82(\mathrm{~m}, 1 \mathrm{H}$, pro- $\alpha), 4.83-4.87$ (m, 1H, Phe-CH), 6.33 (brt, 1H, NH), 7.04-7.07 (m, 1H, ArH), 7.18-7.21 (m, 3H, ArH), 7.25-7.28 (m, 3H, ArH), 7.41-7.44 (m, 2H, ArH), $8.24(\mathrm{~d}, J=9.0 \mathrm{~Hz}, 1 \mathrm{H}, \mathrm{NH}), 8.60$ $(\mathrm{d}, J=8.5 \mathrm{~Hz}, 1 \mathrm{H}, \mathrm{NH}), 11.52$ (brs, $1 \mathrm{H}, \mathrm{NH}) \mathrm{ppm} ;{ }^{13} \mathrm{C}$ $\mathrm{NMR}\left(\mathrm{CDCl}_{3}, 125 \mathrm{MHz}\right) \delta 13.7\left(\mathrm{CH}_{3}\right), 20.2\left(\mathrm{CH}_{2}\right), 25.7$ $\left(\mathrm{CH}_{2}\right), 30.2$ (pro- $\gamma$ ), 31.4 (pro- $\beta$ ), $37.8\left(\right.$ Phe- $\left.\mathrm{CH}_{2}\right), 39.6$ $\left(\mathrm{NHCH}_{2}\right), 46.8$ (pro- $\delta$ ), 54.4 (Phe- $\left.\mathrm{CH}\right), 60.4$ (pro- $\alpha$ ), 121.0 $\left(\mathrm{C}_{\text {aro }} \mathrm{H}\right), 121.2\left(\mathrm{C}_{\text {aro }} \mathrm{H}\right), 123.0\left(\mathrm{C}_{\text {aro }} \mathrm{H}\right), 126.4\left(\mathrm{C}_{\text {aro }} \mathrm{H}\right)$, $126.8\left(\mathrm{C}_{\text {aro }} \mathrm{H}\right), 128.4\left(\mathrm{C}_{\text {aro }} \mathrm{H}\right), 129.3\left(\mathrm{C}_{\text {aro }} \mathrm{H}\right), 132.4\left(\mathrm{C}_{\text {aro }}\right)$, $136.9\left(\mathrm{C}_{\text {aro }}\right), 139.8\left(\mathrm{C}_{\text {aro }}\right), 168.7(\mathrm{C}=\mathrm{O}), 170.1(\mathrm{C}=\mathrm{O}), 175.6$ $(\mathrm{C}=\mathrm{O}) \mathrm{ppm}$; LC-MS (ESI-QTOF) $\mathrm{m} / z[\mathrm{M}+\mathrm{H}]^{+}$, found 437.2638. $\mathrm{C}_{25} \mathrm{H}_{32} \mathrm{~N}_{4} \mathrm{O}_{3}$ requires 437.2553; FTIR (ATR) $v$ $3335,3244,3061,3025,2955,2930,1688,1661,1624,1588$, $1490,1435,1282,1185 \mathrm{~cm}^{-1}$.

(S)-Metyl 2-((S)-3-hydroxy-2-((S)-3-pyrrolidine-2-carboxamido)propanamido)-3-phenylpropanoate (7c)

Colorless oil, yield $90 \%,[\alpha]^{20}=+6.0(\mathrm{c}=1.00$, $\left.\mathrm{CH}_{3} \mathrm{OH}\right) ;{ }^{1} \mathrm{H}$ NMR $\left(\mathrm{CD}_{3} \mathrm{OD}, 500 \mathrm{MHz}\right) \delta 1.86-1.95(\mathrm{~m}$, $3 \mathrm{H}, 2 \times$ pro- $\gamma$ and pro- $\beta), 2.24-2.31(\mathrm{~m}, 1 \mathrm{H}$, pro- $\beta), 3.01-$ $3.05\left(\mathrm{~m}, 1 \mathrm{H}, \mathrm{PhCH}_{2}\right), 3.12-3.20\left(\mathrm{~m}, 3 \mathrm{H}, \mathrm{PhCH}_{2}\right.$ and $2 \times$ pro- $\delta$ ), $3.66\left(\mathrm{~m}, 2 \mathrm{H}, \mathrm{CH}_{2} \mathrm{OH}\right), 3.70\left(\mathrm{~s}, 3 \mathrm{H}, \mathrm{OCH}_{3}\right), 4.01-$ $4.04(\mathrm{~m}, 1 \mathrm{H}$, pro- $\alpha), 4.47-4.49(\mathrm{~m}, 1 \mathrm{H}$, Phe- $\mathrm{CH}), 4.71-$ $4.74\left(\mathrm{~m}, 1 \mathrm{H}, \mathrm{CHCH}_{2} \mathrm{OH}\right), 7.20-7.31(\mathrm{~m}, 5 \mathrm{H}, \mathrm{ArH}) \mathrm{ppm}$; ${ }^{13} \mathrm{C} \mathrm{NMR}\left(\mathrm{CD}_{3} \mathrm{OD}, 125 \mathrm{MHz}\right) \delta 26.1$ (pro- $\left.\gamma\right), 31.5$ (pro- $\beta$ ), $38.4\left(\mathrm{PhCH}_{2}\right), 47.7$ (pro- $\delta$ ), $52.7\left(\mathrm{OCH}_{3}\right), 55.2$ (pro- $\left.\alpha\right)$, $56.4\left(\mathrm{CHCH}_{2} \mathrm{OH}\right), 61.4(\mathrm{Phe}-\mathrm{CH}), 63.1\left(\mathrm{CH}_{2} \mathrm{OH}\right), 128.0$ $\left(\mathrm{C}_{\text {aro }} \mathrm{H}\right), 129.5\left(\mathrm{C}_{\text {aro }} \mathrm{H}\right), 130.3\left(\mathrm{C}_{\text {aro }} \mathrm{H}\right), 137.8\left(\mathrm{C}_{\text {aro }}\right), 171.8$ $(\mathrm{C}=\mathrm{O}), \quad 173.2 \quad(\mathrm{C}=\mathrm{O}), \quad 174.2 \quad(\mathrm{C}=\mathrm{O}) \quad \mathrm{ppm} ; \quad$ LC-MS (ESI-QTOF) $m / z[\mathrm{M}+\mathrm{H}]^{+}$, found $364.1868 . \mathrm{C}_{18} \mathrm{H}_{25} \mathrm{~N}_{3} \mathrm{O}_{5}$ requires 364.1872; FTIR (ATR) v 3340, 2958, 2848, 1666, $1554,1450,1191,1136 \mathrm{~cm}^{-1}$.

General procedure for aldol reaction catalyzed by organocatalyst $7 \mathrm{c}$

The catalyst $7 \mathrm{c}(0.10 \mathrm{mmol})$ and benzoic acid $(0.10$ mmol) were stirred in water at $0{ }^{\circ} \mathrm{C}$ for $10 \mathrm{~min}$. Then, aldehyde $(1.00 \mathrm{mmol})$ and ketone $(10.00 \mathrm{mmol})$ were added, and the reaction mixture was stirred at $0{ }^{\circ} \mathrm{C}$ until the reaction completed. After the evaporation of water, the crude products were purified by column chromatography, eluted by EtOAc/hexane mixture. The enantioselectivity was determined by chiral HPLC with a Chiralpak AD and AD-H columns (UV detection set at $254 \mathrm{~nm}, i-\mathrm{PrOH} /$ hexane as eluent).

\section{Acknowledgement}

We thank Yildiz Technical University Scientific Research Foundation (2015-01-02-YL09) for financial support. 


\section{References}

1. M. M. Heravi, V. Zadsirjan, M. Dehghani, N. Hosseintash, Tetrahedron Asymm. 2017, 28, 587-707.

DOI:10.1016/j.tetasy.2017.04.006

2. G. Guillena, C. Najera, D. J. Ramon, Tetrahedron Asymm. 2007, 18, 2249-2293. DOI:10.1016/j.tetasy.2007.09.025

3. H. Akutsu, K. Nakashima, H. Yanai, A. Kotani, S.-i. Hirashima, T. Yamamoto, R. Takahashi, A. Yoshida, Y. Koseki, H. Hakamata, T. Matsumoto, T. Miura, Synlett 2017, 28, 13631367. DOI:10.1055/s-0036-1558971

4. D. Bhattacharjee, D. Sutradhar, A. K. Chandra, B. Myrboh, Tetrahedron 2017, 73, 3497-3504.

DOI:10.1016/j.tet.2017.05.025

5. A. Yazdani-Elah-Abadi, S. A. Pour, M. Kangani, R. Mohebat, R. Monatsh. Chem. 2017, 148, 2135-2142.

DOI:10.1007/s00706-017-2008-7

6. H. Hegde, N. S. Shetty, Chem. Het. Comp. 2017, 53 (8), 883886. DOI:10.1007/s10593-017-2152-3

7. S. Nagaraju, O. Perumal, P. K. Divakar, B. Paplal, D. Kashinath, New J. Chem. 2017, 41, 8993-9001.

DOI:10.1039/C7NJ01021D

8. M. T. Robak, M. A. Herbage, J. A. Elman, Tetrahedron 2011, 67, 4412-4416. DOI:10.1016/j.tet.2011.03.030

9. P. Milbeo, K. Maurent, L. Moulat, A. Lebrun, C. Didierjean, E. Aubert, J. Martinez, M. Calmes, Tetrahedron 2016, 72, 17061713. DOI:10.1016/j.tet.2016.02.027

10. E. Machuca, E. Juaristi, Tetrahedron Lett. 2015, 56, 11441148. DOI:10.1016/j.tetlet.2015.01.079

11. Z. Y. Li, Y. Chen, C. Q. Zheng, Y. Yin, L. Wang, X. Q. Sun, Tetrahedron 2017, 73, 78-85.

DOI:10.1016/j.tet.2016.11.052

12. O. Illa, O. Porcar-Tost, C. Robledillo, C. Elvira, P. Nolis, O. Reiser, V. Branchadell, R. M. Ortuno, J. Org. Chem. 2018, 83, 350-363. DOI:10.1021/acs.joc.7b02745

13. Q. Li, Y. Li, J. Wang, Y. Lin, Z. Wei, H. Duan, Q. Yang, F. Bai, Y. Li, New J. Chem. 2018, 42, 827-831.

DOI:10.1039/C7NJ03912C

14. A. O. Zuniga, M. Milan, E. Juaristi, Org. Lett. 2017, 19, $1108-$ 1111. DOI:10.1021/acs.orglett.7b00129

15. T. P. Kumar, R. C. Shekhar, K. S. Sunder, R. Vadaparthi, Tetrahedron Asymm. 2015, 26, 543-547.

DOI:10.1016/j.tetasy.2015.03.009

16. E. Machuca, G. Granados, B. Hinajosa, E. Juaristi, Tetrahedron Lett. 2015, 56, 6047-6051.

DOI:10.1016/j.tetlet.2015.09.062

17. L. L. Lou, J. Zhang, H. Du, B. Zhao, S. Li, W. Yu, K. Yu, S. Liu, Catal. Today. 2016, 264, 109-114.

DOI:10.1016/j.cattod.2015.10.004

18. T. P. Kumar, N. Manjula, K. Katragunta, Tetrahedron Asymm. 2015, 26, 1281-1284. DOI:10.1016/j.tetasy.2015.09.018

19. G. Guo, Y. Wu, X. Zhao, J. Wang, L. Zhang, Y. Cui, Tetrahedron Asymm. 2016, 27, 740-746.

DOI:10.1016/j.tetasy.2016.06.014

20. Y. Zhou, G. Yang, C. Lu, J. Nie, Z. Chen, J. Ren, Catal.Commun. 2016, 75, 23-27. DOI:10.1016/j.catcom.2015.11.017
21. W. Zhao, C. Qu, L. Yang, Y. Cui, Chinese J. Catal., 2015, 36, 367-371. DOI:10.1016/S1872-2067(14)60248-5

22. G. D. Yadav, S. Singh, Tetrahedron Asymm. 2016, 27, 123-129. DOI:10.1016/j.tetasy.2015.12.005

23. E.-J. Xu, Y. Song, Z.-L. Wei, R. Wang, H.-F. Duan, Y.-J. Lin, Q-B. Yang, Y.-X. Li, Can. J. Chem. 2019, 97, 352-359.

DOI:10.1139/cjc-2018-0352

24. N. Mase, Y. Nakai, N. Ohara, H. Yoda, K. Takabe, F. Tanaka, C. F. Barbas III, J. Am. Chem. Soc. 2006, 128, 734-735. DOI:10.1021/ja0573312

25. M. Lei, L. Shi, G. Li, S. Chen, W. Fang, Z. Ge, T. Cheng, R. Li, Tetrahedron 2007, 63, 7892-7898.

DOI:10.1016/j.tet.2007.05.077

26. A. Ahmetlli, N. Spiliopoulou, A. Magi-Oikonomopoulou, D.T. Gerokonstantis, P. Moutevelis-Minakakis, C. G. Kokotos, Tetrahedron 2018, 74, 5987-5995.

DOI:10.1016/j.tet.2018.08.038

27. Y.-H. Chen, P.-H. Sung, K. Sung, Amino Acids 2010, 38, 839845. DOI:10.1007/s00726-009-0290-3

28. Z. Tang, Z.-H. Yang, L.-F. Cun, L.-Z. Gong, A.-Q. Mi, Y.-Z. Jiang, Org. Lett. 2004, 6, 2285-2287.

DOI:10.1021/ol049141m

29. P. Krattiger, R. Kovasy, J. D. Revell, S. Ivan, H. Wennemers, Org. Lett. 2005, 7, 1101-1103. DOI:10.1021/ol0500259

30. J. D. Revell, H. Wennemers, Adv. Synth. Catal. 2008, 350, 1046-1052. DOI:10.1002/adsc.200800053

31. M. Orlandi, M. Benaglia, L. Raimondi, G. Celentano, G. Eur. J. Org. Chem. 2013, 2346-2354.

DOI:10.1002/ejoc.201201643

32. I. Vlasserou, M. Sfetsa, D. T. Gerokonstantis, C. G. Kokotos, P. Moutevelis-Minakakis, Tetrahedron 2018, 74, 2338-2349. DOI:10.1016/j.tet.2018.03.054

33. T. Yorulmaz, F. Aydogan, C. Yolacan, Synth. Commun. 2017, 47, 78-85. DOI:10.1080/00397911.2016.1252988

34. C. Yolacan, M. E. Mavis, F. Aydogan, Tetrahedron 2014, 70, 3707-3713. DOI:10.1016/j.tet.2014.04.016

35. A. Ozkan, M. E. Mavis, F. Aydogan, C. Yolacan, J. Chem. Soc. Pak. 2014, 36, 326-331.

36. M. Cicek, F. Aydogan, C. Yolacan, Lett. Org. Chem. 2017, 14, 778-786. DOI:10.2174/1570178614666170724125842

37. J. Priego, P. Flores, C. Ortiz-Nava, J. Escalate, Tetrahedron Asymm. 2004, 15, 3545-3549.

DOI:10.1016/j.tetasy.2004.08.032

38. M. M. Zhang, L. Lu, X. S. Wang, J. Het. Chem. 2014, 51, 13631368. DOI: $10.1002 /$ jhet. 1826

39. J. Zhao, L. He, J. Jiang, Z. Tang, L.-F. Cun, L.-Z. Gong, Tetrahedron Lett. 2008, 49, 3372-3375.

DOI:10.1016/j.tetlet.2008.03.131

40. S.-D. Fu, X.-K. Fu, S.-P. Zhang, X.-C. Zou, X.-J. Wu, Tetrahedron Asymmetry. 2009, 20, 2390-2396.

DOI:10.1016/j.tetasy.2009.09.019

41. Q. Zhao, Y.-H. Lam, M. Kheirabadi, C. Xu, K. N. Houk, C. E. Schafmeister, J. Org. Chem. 2012, 77, 4784-4792.

DOI:10.1021/jo300569c

42. V. Gauchot, A. R. Schmitzer, J. Org. Chem. 2012, 77, 49174923. DOI:10.1021/jo300737u 
43. M. Lei, L. Shi, G. Li, S. Chen, W. Fang, Z. Ge, T. Cheng, R. Li, Tetrahedron. 2007, 63, 7892-7898.

DOI:10.1016/j.tet.2007.05.077

44. C. A. Thomas, E. R. Talaty, J. G. Bann, Chem. Commun. 2009, 3366-3368. DOI:10.1039/b821952d

45. S. Chaudhary, H. Kumar, H. Verma, A. Rajpoot, Int. J. Pharm Tech Res. 2012, 4, 194-200.
46. Z. Chen, J. Deng, T. Ye, Arkivoc 2003, 7, 268-285. DOI:10.3998/ark.5550190.0004.723

47. S. Cheng, X. Zhang, W. Wang, M. Zhao, M. Zheng, H. W. Chang, J. Wu, S. Peng, Eur. J. Med. Chem. 2009, 44, 49044919. DOI:10.1016/j.ejmech.2009.08.002

48. B. McKeever, G. Pattenden, Tetrahedron 2003, 59, 2713-2727. DOI:10.1016/S0040-4020(03)00294-1

\section{Povzetek}

Prispevek poroča o sintezi novih di- in triamidnih organokatalizatorjih, pridobljenih iz (L)-prolina, in njihovi uspešni uporabi v neposredni asimetrični aldolni kondenzaciji alifatskih ketonov in aromatskih aldehidov v vodi pri $0{ }^{\circ} \mathrm{C} \mathrm{v}$ prisotnosti benzojske kisline kot ko-katalizatorja. (S)-metil-2-((S)-3-hidroksi-2-((S)-3-pirolidin-2-karboksamido)propanamido)-3-fenilpropanoat (7c) je kot organokatalizator pri teh reakcijskih pogojih pokazal najboljše rezultate $\mathrm{z}$ dobro diastereoselektivnostjo (do 99 \%), enantioselektivnostjo (do 98 \%) in izkoristkom reakcij (do 91 \%). 